\title{
The new $(g-2)_{\mu}$ result and the $\mu \nu \mathrm{SSM}$
}

\author{
Sven Heinemeyer ${ }^{1,2,3, a}$, Essodjolo Kpatcha ${ }^{1,4, b}$, Iñaki Lara ${ }^{5, c}$, Daniel E. López-Fogliani ${ }^{6,7, d}$, Carlos Muñoz ${ }^{1,4, e}$, \\ Natsumi Nagata ${ }^{8, f}$ \\ ${ }^{1}$ Instituto de Física Teórica (IFT) UAM-CSIC, Campus de Cantoblanco, 28049 Madrid, Spain \\ ${ }^{2}$ Campus of International Excellence UAM + CSIC, Cantoblanco, 28049 Madrid, Spain \\ ${ }^{3}$ Instituto de Física de Cantabria (CSIC-UC), 39005 Santander, Spain \\ ${ }^{4}$ Departamento de Física Teórica, Universidad Autónoma de Madrid (UAM), Campus de Cantoblanco, 28049 Madrid, Spain \\ ${ }^{5}$ Faculty of Physics, University of Warsaw, Pasteura 5, 02-093 Warsaw, Poland \\ ${ }^{6}$ Departamento de Física, Facultad de Ciencia Exactas y Naturales, Instituto de Física de Buenos Aires UBA and CONICET, Universidad de \\ Buenos Aires, 1428 Buenos Aires, Argentina \\ ${ }^{7}$ Pontificia Universidad Católica Argentina, Av. Alicia Moreau de Justo 1500, 1107 Buenos Aires, Argentina \\ ${ }^{8}$ Department of Physics, University of Tokyo, Tokyo 113-0033, Japan
}

Received: 25 April 2021 / Accepted: 30 August 2021 / Published online: 9 September 2021

(C) The Author(s) 2021

\begin{abstract}
The $\mu \nu \mathrm{SSM}$ is a highly predictive alternative model to the MSSM. In particular, the electroweak sector of the model can explain the longstanding discrepancy between the experimental result for the anomalous magnetic moment of the muon, $(g-2)_{\mu}$, and its Standard Model prediction, while being in agreement with all other theoretical and experimental constraints. The recently published MUON G-2 result is within $0.8 \sigma$ in agreement with the older BNL result on $(g-2)_{\mu}$. The combined result was announced as $a_{\mu}^{\exp }=(11659206.1 \pm 4.1) \times 10^{-10}$, yielding a new deviation from the Standard Model prediction of $\Delta a_{\mu}=(25.1 \pm 5.9) \times 10^{-10}$, corresponding to $4.2 \sigma$. Using this improved bound we update the analysis in the $\mu \nu \mathrm{SSM}$ as presented in Kpatcha et al. (Eur Phys J C 81(2):154. arXiv:1912.04163 [hep-ph], 2021) and set new limits on the allowed parameters space of the electroweak sector of the model. We conclude that significant regions of the model can explain the new $(g-2)_{\mu}$ data.
\end{abstract}

\section{Introduction}

The experimental result for the anomalous magnetic moment of the muon, $a_{\mu}:=\frac{1}{2}(g-2)_{\mu}$, was so far dominated by

\footnotetext{
a e-mail: Sven.Heinemeyer@cern.ch

b e-mail: kpatcha.essodjolo@uam.es

c e-mail: inaki.lara@fuw.edu.pl

de-mail: daniel.lopez@df.uba.ar

e e-mail: c.munoz@uam.es (corresponding author)

fe-mail: natsumi@hep-th.phys.s.u-tokyo.ac.jp
}

the measurements made at Brookhaven National Laboratory (BNL) [2], resulting in a world average of [3]

$a_{\mu}^{\exp -\mathrm{BNL}}=(11659209.1 \pm 6.3) \times 10^{-10}$,

combining statistical and systematic uncertainties. The Standard Model (SM) prediction of $a_{\mu}$ is given by [4] (based on Refs. [5-24]),

$a_{\mu}^{\mathrm{SM}}=(11659181.0 \pm 4.3) \times 10^{-10}$,

corresponding to a $3.7 \sigma$ discrepancy.

Recently, the MUON G-2 collaboration [25] published the results (referred to as "FNAL" result) of their Run 1 data [26,27],

$a_{\mu}^{\text {exp-FNAL }}=(11659204.0 \pm 5.4) \times 10^{-10}$,

being within $0.8 \sigma$ well compatible with the previous experimental result in Eq. (1). The combined experimental result was announced as

$a_{\mu}^{\exp }=(11659206.1 \pm 4.1) \times 10^{-10}$.

Compared with the SM prediction in Eq. (2), this yields a new deviation of

$\Delta a_{\mu}=(25.1 \pm 5.9) \times 10^{-10}$,

corresponding to a $4.2 \sigma$ discrepancy. ${ }^{1}$

\footnotetext{
1 A recent calculation in lattice QCD of the contribution of the hadronic vacuum polarization [28], results in that there is no essential discrepancy
} 
In Ref. [1], some of us performed an analysis of the (then current) deviation of $a_{\mu}[5,6]$, taking into account all relevant data for the electroweak (EW) sector of the ' $\mu$ from $\nu$ ' Supersymmetric Standard Model ( $\mu \nu \mathrm{SSM}$ ) [33] (for a recent review of the model, see Ref. [34]). The experimental results imposed comprise (as will be detailed in Sect. 2) Higgs and neutrino data, flavor observables such as $B$ and $\mu$ decays, as well as the direct searches at the LHC [35-42]. Sampling the $\mu \nu \mathrm{SSM}$, it was analyzed which parameter (combinations) are favored by $a_{\mu}$ measurements. It was found that the $\mu \nu \mathrm{SSM}$ can naturally produce moderately light left-handed muonsneutrinos $\left(120 \mathrm{GeV} \lesssim m_{\widetilde{v}_{\mu}} \lesssim 620 \mathrm{GeV}\right)$ and wino-like charginos $\left(200 \mathrm{GeV} \lesssim m_{\widetilde{W}^{ \pm}} \lesssim 930 \mathrm{GeV}\right.$ ), accommodating the discrepancy between experimental and SM values. A recent general analysis in the Minimal Supersymmetric Standard Model (MSSM) [43-47] can be found in Refs. [48,49].

In this work, we analyze the combination of the FNAL Run 1 data with the previous BNL result [26,27]. Using this improved bound we update the results presented in Ref. [1] and set new limits on the allowed parameters space of the EW sector of the $\mu \nu \mathrm{SSM}$, as shown in Figs. 8 and 9 to be discussed below. The results will be discussed in the context of searches for EW particles at the LHC and future colliders. A recent general analysis of the impact of the new FNAL result in the MSSM can be found in Ref. [50].

\section{The model and the experimental constraints}

\subsection{The $\mu \nu \mathrm{SSM}$ and $a_{\mu}$}

In the $\mu \nu$ SSM $[33,34]$, the particle content of the MSSM is extended by right-handed neutrino superfields $\hat{v}_{i}^{c}$. The simplest superpotential of the model is the following $[33,51,52]$ :

$$
\begin{aligned}
W= & \epsilon_{a b}\left(Y_{e_{i j}} \hat{H}_{d}^{a} \hat{L}_{i}^{b} \hat{e}_{j}^{c}+Y_{d_{i j}} \hat{H}_{d}^{a} \hat{Q}_{i}^{b} \hat{d}_{j}^{c}+Y_{u_{i j}} \hat{H}_{u}^{b} \hat{Q}^{a} \hat{u}_{j}^{c}\right) \\
& +\epsilon_{a b}\left(Y_{v_{i j}} \hat{H}_{u}^{b} \hat{L}_{i}^{a} \hat{v}_{j}^{c}-\lambda_{i} \hat{v}_{i}^{c} \hat{H}_{u}^{b} \hat{H}_{d}^{a}\right)+\frac{1}{3} \kappa_{i j k} \hat{v}_{i}^{c} \hat{v}_{j}^{c} \hat{v}_{k}^{c},
\end{aligned}
$$

where the summation convention is implied on repeated indices, with $i, j, k=1,2,3$ the usual family indices of the SM and $a, b=1,2 S U(2)_{L}$ indices with $\epsilon_{a b}$ the totally antisymmetric tensor, $\epsilon_{12}=1$.

Working in the framework of a typical low-energy supersymmetry (SUSY), the Lagrangian containing the soft SUSY-breaking terms related to $W$ is given by:

with the experimental data. However, the authors of Ref. [29] have argued that the estimation of the uncertainty of the lattice calculation is too optimistic. In addition, several authors have pointed out that such a result implies a tension with electroweak fits [30-32].

$$
\begin{aligned}
-\mathcal{L}_{\mathrm{soft}}= & \epsilon_{a b}\left(T_{e_{i j}} H_{d}^{a} \widetilde{L}_{i L}^{b} \widetilde{e}_{j R}^{*}+T_{d_{i j}} H_{d}^{a} \widetilde{Q}_{i L}^{b} \widetilde{d}_{j R}^{*}\right. \\
& \left.+T_{u_{i j}} H_{u}^{b} \widetilde{Q}_{i L}^{a} \widetilde{u}_{j R}^{*}+\text { h.c. }\right) \\
& +\epsilon_{a b}\left(T_{v_{i j}} H_{u}^{b} \widetilde{L}_{i L}^{a} \widetilde{v}_{j R}^{*}-T_{\lambda_{i}} \widetilde{v}_{i R}^{*} H_{d}^{a} H_{u}^{b}\right. \\
& \left.+\frac{1}{3} T_{\kappa_{i j k}} \widetilde{v}_{i R}^{*} \widetilde{v}_{j R}^{*} \widetilde{v}_{k R}^{*}+\text { h.c. }\right) \\
& +m_{\widetilde{Q}_{i j L}}^{2} \widetilde{Q}_{i L}^{a *} \widetilde{Q}_{j L}^{a}+m_{\widetilde{u}_{i j R}}^{2} \widetilde{u}_{i R}^{*} \widetilde{u}_{j R}+m_{\widetilde{d}_{i j R}}^{2} \widetilde{d}_{i R}^{*} \widetilde{d}_{j R} \\
& +m_{\widetilde{L}_{i j L}}^{2} \widetilde{L}_{i L}^{a *} \widetilde{L}_{j L}^{a}+m_{\widetilde{v}_{i j R}}^{2} \widetilde{v}_{i R}^{*} \widetilde{v}_{j R} \\
& +m_{\widetilde{e}_{i j R}}^{2} \widetilde{e}_{i R}^{*} \widetilde{e}_{j R}+m_{H_{d}}^{2} H_{d}^{a *} H_{d}^{a}+m_{H_{u}}^{2} H_{u}^{a *} H_{u}^{a} \\
& +\frac{1}{2}\left(M_{3} \widetilde{g} \widetilde{g}^{2}+M_{2} \widetilde{W}_{W} \widetilde{W}_{1} M_{1} \widetilde{B}^{0} \widetilde{B}^{0}+\text { h.c. }\right)
\end{aligned}
$$

In case of following the usual assumption based on the breaking of supergravity, that all the soft trilinear parameters are proportional to their corresponding couplings in the superpotential (for a review, see e.g. Ref. [53]), one can write

$T_{e_{i j}}=A_{e_{i j}} Y_{e_{i j}}, \quad T_{d_{i j}}=A_{d_{i j}} Y_{d_{i j}}, \quad T_{u_{i j}}=A_{u_{i j}} Y_{u_{i j}}$

$T_{v_{i j}}=A_{v_{i j}} Y_{v_{i j}}, \quad T_{\lambda_{i}}=A_{\lambda_{i}} \lambda_{i}, \quad T_{\kappa_{i j k}}=A_{\kappa_{i j k}} \kappa_{i j k}$,

where the summation convention on repeated indices does not apply.

In the early universe not only the EW symmetry is broken, but in addition to the neutral components of the Higgs doublet fields $H_{d}$ and $H_{u}$ also the left-handed (LH) and righthanded (RH) scalar neutrinos $\widetilde{v}_{i}$ and $\widetilde{v}_{i R}$ acquire a vacuum expectation value (vev). With the choice of CP conservation, they develop real vevs denoted by:

$$
\left\langle H_{d}^{0}\right\rangle=\frac{v_{d}}{\sqrt{2}}, \quad\left\langle H_{u}^{0}\right\rangle=\frac{v_{u}}{\sqrt{2}}, \quad\left\langle\widetilde{v}_{i R}\right\rangle=\frac{v_{i R}}{\sqrt{2}}, \quad\left\langle\widetilde{v}_{i}\right\rangle=\frac{v_{i}}{\sqrt{2}} .
$$

The EW symmetry breaking is induced by the scalar and gaugino soft SUSY-breaking masses and soft SUSY-breaking trilinear parameters $A$, which are all of $\mathcal{O}(1 \mathrm{TeV})$, and therefore also $v_{i R} \sim \mathcal{O}(1 \mathrm{TeV})$ as a consequence of their minimization equations in the scalar potential $[33,51,52]$. Since $\widetilde{v}_{i R}$ are gauge-singlet fields, the $\mu$-problem can be solved in total analogy to the Next-to-MSSM (NMSSM) [54,55] through the presence in the superpotential (6) of the trilinear terms $\lambda_{i} \hat{v}_{i}^{c} \hat{H}_{d} \hat{H}_{u}$. Then, the value of the effective $\mu$-parameter is given by $\mu=\lambda_{i} v_{i R} / \sqrt{2}$. These trilinear terms also relate the origin of the $\mu$-term to the origin of neutrino masses and mixing angles, since the Yukawa couplings $Y_{v_{i j}} \hat{H}_{u} \hat{L}_{i} \hat{v}_{j}^{c}$ are present in the superpotential generating Dirac masses for neutrinos, $m_{\mathcal{D}_{i j}} \equiv Y_{v_{i j}} v_{u} / \sqrt{2}$. Remark- 
ably, in the $\mu \nu \mathrm{SSM}$ it is possible to accommodate neutrino masses and mixings in agreement with experiments [56-59], via an EW seesaw mechanism dynamically generated during the EW symmetry breaking [33,51,60-64] through the couplings $\kappa_{i j k} \hat{v}_{i}^{c} \hat{v}_{j}^{c} \hat{v}_{k}^{c}$ giving rise to effective Majorana masses for RH neutrinos, $\mathcal{M}_{i j}=2 \kappa_{i j k} \frac{v_{k R}}{\sqrt{2}}$. Actually, this is possible at tree level even with diagonal Yukawa couplings [60,62], i.e. $Y_{v_{i j}}=Y_{v_{i}} \delta_{i j}$.

Therefore, the $\mu \nu \mathrm{SSM}$ solves the $\mu$ - and the $\nu$-problem (neutrino masses) simultaneously without the need to introduce additional energy scales beyond the SUSY-breaking scale. In contrast to the (N)MSSM, $R$-parity and lepton number are not conserved, leading to a completely different phenomenology characterized by distinct prompt or displaced decays of the lightest supersymmetric particle (LSP), producing multi-leptons/jets/photons with small/moderate missing transverse energy (MET) from neutrinos [52,65-67]. The low decay width of the LSP due to the smallness of neutrino masses is also related to the existence of possible candidates for decaying dark matter in the model. This is the case of the gravitino [68-72], or the axino [73], with lifetimes greater than the age of the Universe. It is also worth mentioning concerning cosmology, that baryon asymmetry might be realized in the $\mu \nu$ SSM through EW baryogenesis [74]. The present limit of the electric dipole moment (EDM) of the electron highly constrains the EW baryogenesis scenario (for a review see e.g. Ref. [75]), but since in the $\mu \nu$ SSM there are many scalar fields, EW baryogenesis with "multi-step phase transition" may allow successful baryogenesis while evading the EDM bound. This analysis needs a dedicated study, which would be very interesting to realize in the future.

The parameter space of the $\mu \nu \mathrm{SSM}$, and in particular the neutrino, neutral Higgs, slepton, chargino and neutralino sectors are relevant for our analysis in order to reproduce neutrino, Higgs and $a_{\mu}$ data. They were discussed in Ref. [1], and we refer the reader to that work for details. Here we summarize the analysis. Using diagonal mass matrices for the scalar fermions, in order to avoid the strong upper bounds upon the intergenerational scalar mixing (see e.g. Ref. [76]), from the eight minimization conditions with respect to $v_{d}, v_{u}, v_{i R}$ and $v_{i L}$ to facilitate the computation we prefer to eliminate the soft masses $m_{H_{d}}^{2}, m_{H_{u}}^{2}, m_{\widetilde{v}_{i j R}}^{2}$ and $m_{\widetilde{L}_{i j L}}^{2}$ in favor of the VEVs. Also, we assume for simplicity in what follows that the couplings $\lambda_{i}=\lambda, \kappa_{i j k}=\kappa \delta_{i j} \delta_{j k}$, and the vevs $v_{i R}=v_{R}$. Then, the higgsino mass parameter $\mu$, and Dirac and Majorana masses discussed above are given by:

$\mu=3 \lambda \frac{v_{R}}{\sqrt{2}}, \quad m_{\mathcal{D}_{i}}=Y_{v_{i}} \frac{v_{u}}{\sqrt{2}}, \quad \mathcal{M}=2 \kappa \frac{v_{R}}{\sqrt{2}}$

For the light neutrinos, under the above assumption, one can obtain the following simplified formula for the effective mass matrix [62]: $\left(m_{v}\right)_{i j} \approx \frac{m_{\mathcal{D}_{i}} m_{\mathcal{D}_{j}}}{3 \mathcal{M}}\left(1-3 \delta_{i j}\right)-\frac{v_{i} v_{j}}{4 M}, \quad \frac{1}{M} \equiv \frac{g^{\prime 2}}{M_{1}}+\frac{g^{2}}{M_{2}}$,

where $g^{\prime}, g$ are the EW gauge couplings, and $M_{1}, M_{2}$ the bino and wino soft SUSY-breaking masses, respectively. This expression arises from the generalized EW seesaw of the $\mu \nu \mathrm{SSM}$, where due to $R$-parity violation (RPV) the neutral fermions have the flavor composition $\left(v_{i}, \widetilde{B}^{0}, \widetilde{W}^{0}, \widetilde{H}_{d}^{0}, \widetilde{H}_{u}^{0}, v_{i R}\right)$. Of the three terms in Eq. (11), the first two are generated through the mixing of $v_{i}$ with $v_{i R^{-}}$ Higgsinos, and the third one also include the mixing with the gauginos. These are the so-called $v_{R}$-Higgsino seesaw and gaugino seesaw, respectively [62]. One can see from this equation that once $\mathcal{M}$ is fixed, as will be done in the parameter analysis of Sect. 3, the most crucial independent parameters determining neutrino physics are:

$Y_{v_{i}}, v_{i}, M_{1}, M_{2}$.

Note that this EW scale seesaw implies $Y_{v_{i}} \lesssim 10^{-6}$ driving $v_{i}$ to small values because of the proportional contributions to $Y_{v_{i}}$ appearing in their minimization equations. A rough estimation gives $v_{i} \lesssim m_{\mathcal{D}_{i}} \lesssim 10^{-4}$.

Considering the normal ordering for the neutrino mass spectrum, and taking advantage of the dominance of the gaugino seesaw for some of the three neutrino families, representative solutions for neutrino physics using diagonal neutrino Yukawas were obtained in Ref. [67]. In particular, the so-called type 3 solutions, which have the structure

$M>0$, with $Y_{v_{2}}<Y_{v_{1}}<Y_{\nu_{3}}$, and $v_{1}<v_{2} \sim v_{3}$,

are especially interesting for our analysis of $a_{\mu}$, since, as will be argued below, they are able to produce the LH muonsneutrino as the lightest of all sneutrinos. In this case of type 3 , it is easy to find solutions with the gaugino seesaw as the dominant one for the second family. Then, $v_{2}$ determines the corresponding neutrino mass and $Y_{\nu_{2}}$ can be small. On the other hand, the normal ordering for neutrinos determines that the first family dominates the lightest mass eigenstate implying that $Y_{v_{1}}<Y_{v_{3}}$ and $v_{1}<v_{2}, v_{3}$, with both $v_{R}$-Higgsino and gaugino seesaws contributing significantly to the masses of the first and third family. Taking also into account that the composition of the second and third families in the third mass eigenstate is similar, we expect $v_{3} \sim v_{2}$.

The LH sneutrinos are mixed with the $\mathrm{RH}$ sneutrinos and neutral Higgses, since the neutral scalars and pseudoscalars in the $\mu \nu \mathrm{SSM}$ have the flavor composition $\left(H_{d}^{0}, H_{u}^{0}, \widetilde{v}_{i R}, \widetilde{v}_{i}\right)$. Nevertheless, the LH sneutrinos are basically decoupled from the other states, since the off-diagonal terms of the mass matrix are suppressed by the small $Y_{v}$ and $v_{i L}$. In addition, scalars have degenerate masses with pseudoscalars $m_{\widetilde{v}_{i}^{\mathcal{R}}} \approx$ $m_{\widetilde{v}_{i}^{\mathcal{I}}} \equiv m_{\widetilde{v}_{i}}$. Given that $m_{\widetilde{L}_{i L}}$ is determined for the three 
Fig. $1 \Delta m_{21}^{2}$ versus neutrino Yukawas (left) and LH sneutrino VEVs (right). Colors blue, green and grey correspond to $i=1,2,3$, respectively
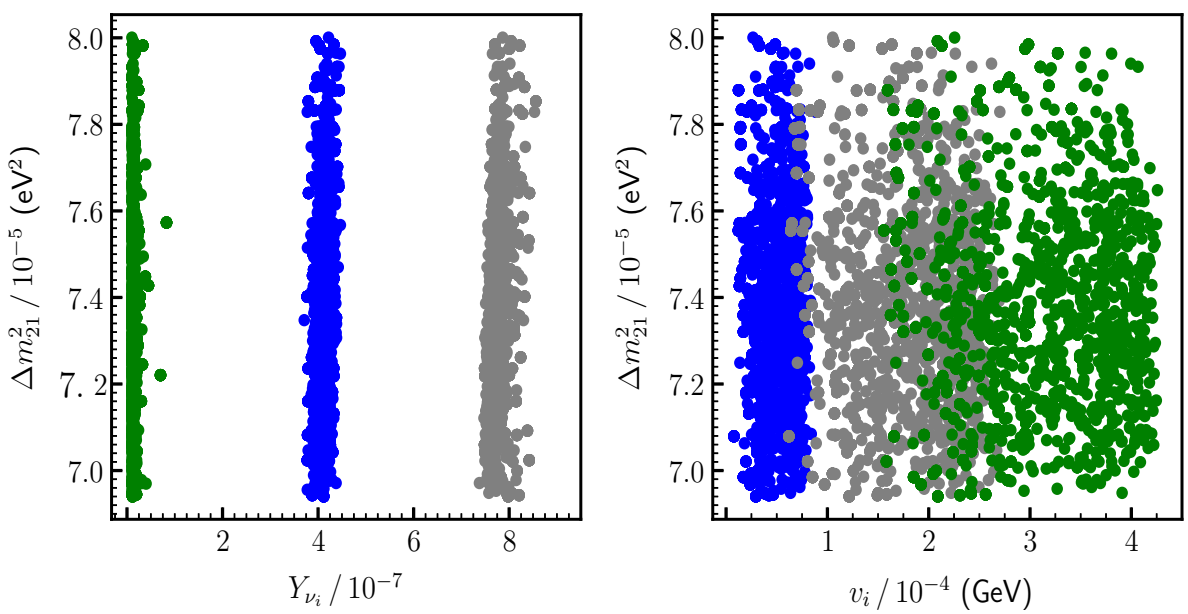

generations from their minimization equations, as discussed above, one arrives to the following approximate tree-level expression for the three LH sneutrino masses $[51,52,60]$ :

$m_{\widetilde{v}_{i}}^{2} \approx \frac{m_{\mathcal{D}_{i}}}{v_{i}} v_{R}\left(\frac{-T_{v_{i}}}{Y_{v_{i}}}-\frac{\mathcal{M}}{2}+\frac{\mu}{\tan \beta}\right)$,

where we have assumed for simplicity that for all soft trilinear parameters $T_{i j}=T_{i} \delta_{i j}$.

As we can see from Eq. (14), the LH sneutrino masses depend not only on LH sneutrino vevs but also on neutrino Yukawas, and as a consequence neutrino physics is very relevant for them. For example, if we work with expression (8) with $A_{v_{i}}$ of $\mathcal{O}(1 \mathrm{TeV})$, neutrino physics determines sneutrino masses through the prefactor $Y_{v_{i}} v_{u} / v_{i}$. Thus, values of this prefactor in the range of about $0.01-1$, i.e. $Y_{v_{i}} \sim 10^{-8}$ $10^{-6}$, will give rise to $\mathrm{LH}$ sneutrino masses in the range of about 100-1000 GeV. This implies that with the hierarchy of neutrino Yukawas $Y_{v_{2}} \sim 10^{-8}-10^{-7}<Y_{v_{1,3}} \sim 10^{-6}$, we can obtain a $\widetilde{v}_{\mu}$ with a mass around $100 \mathrm{GeV}$ whereas the masses of $\widetilde{v}_{e, \tau}$ are of the order of the TeV, i.e. we have $m_{\widetilde{v}_{2}}$ as the smallest of all the sneutrino masses. Clearly, we are in the case of solutions for neutrino physics of type 3 discussed in Eq. (13).

In Fig. 1 from Ref. [1], we show $\Delta m_{21}^{2}=m_{2}^{2}-m_{1}^{2}$ versus $Y_{v_{i}}$ and $v_{i}$ for the scan carried out in that work, using the results for normal ordering from Ref. [59]. As we can see, one can obtain the hierarchy qualitatively discussed above, i.e. $Y_{v_{2}}<Y_{v_{1}}<Y_{v_{3}}$, and $v_{1}<v_{3} \lesssim v_{2}$. We will carry out a similar analysis in Sect. 4 , to correctly reproduce neutrino physics.

Concerning charged sleptons, in the $\mu \nu \mathrm{SSM}$ LH and RH sleptons $\tilde{\ell}_{i L, R}$ are mixed with the charged Higgses, but once again the mixing terms are suppressed and both sectors are essentially decoupled. The same comment applies to the mixing between $\tilde{\ell}_{i L}$ and $\tilde{\ell}_{i R}$ themselves, especially those of the first and second generation because the mixing terms are suppressed by $Y_{e_{i j}}$. In particular, $m_{\tilde{\mu}_{L}}$ and $m_{\tilde{\mu}_{R}}$, which are relevant for our analysis of $a_{\mu}$, are effectively determined by their soft SUSY-breaking masses $m_{\tilde{L}_{2 L}}$ and $m_{\widetilde{e}_{2 R}}$.

Besides, the masses of $\widetilde{v}_{\mu}$ and $\widetilde{\mu}_{L}$ are very similar since these two particles are in the same $S U(2)$ doublet, and therefore both masses are determined by $m_{\tilde{L}_{2 L}}$. At the end of the day, $m_{\tilde{\mu}_{L}}$ is only slightly larger than $m_{\widetilde{v}_{\mu}}$ due to the mass splitting produced by the corresponding $D$-term contributions, $m_{\widetilde{\mu}_{L}}^{2}=m_{\widetilde{v}_{\mu}}^{2}-m_{W}^{2} \cos 2 \beta$.

Therefore, slepton physics introduces two other independent parameters which are relevant for the value of $a_{\mu}$ :

$T_{v_{i}}, m_{\tilde{e}_{2 R}}$

Unlike the LH sneutrinos, the other neutral scalars can be substantially mixed. Neglecting this mixing between the doublet-like Higgses and the three RH sneutrinos, the expression of the tree-level mass of the SM-like Higgs is [51]:

$m_{h}^{2} \approx m_{Z}^{2}\left(\cos ^{2} 2 \beta+10.9 \lambda^{2} \sin ^{2} 2 \beta\right)$

where $\tan \beta=v_{u} / v_{d}$, and $m_{Z}$ denotes the mass of the $Z$ boson. Effects lowering (raising) this mass appear when the SM-like Higgs mixes with heavier (lighter) RH sneutrinos. The one-loop corrections are basically determined by the third-generation soft SUSY-breaking parameters $m_{\tilde{u}_{3 R}}$, $m_{Q_{3 L}}$ and $T_{u_{3}}$. These three parameters together with the coupling $\lambda$ and $\tan \beta$, are the crucial ones for Higgs physics. The colored sector is assumed to be heavy and thus beyond the (current) reach of the LHC. This also ensures that the model can contain a scalar boson with a mass around $\sim 125 \mathrm{GeV}$ and properties similar to the ones of the SM Higgs boson [77-80].

In addition, $\kappa, v_{R}$ and the trilinear parameter $T_{\kappa}$ in the soft Lagrangian (7), are the key ingredients to determine the mass scale of the RH sneutrinos [51,60]. For example, for $\lambda \lesssim 0.01$ they are basically free from any doublet admixture, and using their minimization equations in the scalar potential the scalar and pseudoscalar masses can be approximated respectively by $[52,81]$ : 
Fig. 2 Chargino-LH muon sneutrino (left) and neutralino-smuon (right) one-loop contributions to the anomalous magnetic moment of the muon
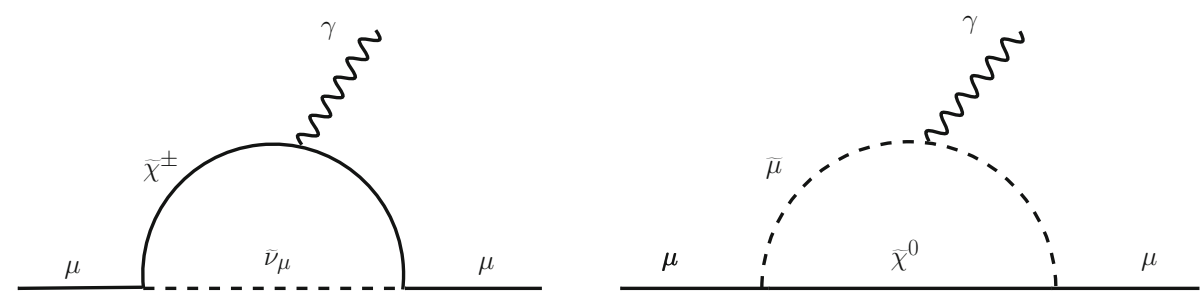

$m_{\widetilde{v}_{i R}^{\mathcal{R}}}^{2} \approx \frac{v_{R}}{\sqrt{2}}\left(T_{\kappa}+\frac{v_{R}}{\sqrt{2}} 4 \kappa^{2}\right), \quad m_{\widetilde{v}_{i R}^{\mathcal{I}}}^{2} \approx-\frac{v_{R}}{\sqrt{2}} 3 T_{\kappa}$.

Finally, $\lambda$ and the trilinear parameter $T_{\lambda}$ not only contribute to these masses for larger values of $\lambda$, but also control the mixing between the singlet and the doublet states and hence, they contribute in determining their mass scales as discussed in detail in Ref. [79]. We conclude that the relevant lowenergy parameters in the Higgs-RH sneutrino sector are:

$\lambda, \kappa, \tan \beta, v_{R}, T_{\kappa}, T_{\lambda}, T_{u_{3}}, m_{\widetilde{u}_{3 R}}, m_{\widetilde{Q}_{3 L}}$.

\subsubsection{SUSY contributions to $a_{\mu}$}

We now turn to the SUSY contributions to $a_{\mu}$. The main contribution to $a_{\mu}$ at the one-loop level in the $\mu \nu \mathrm{SSM}, a_{\mu}^{\mu \nu \mathrm{SSM}}$, comes from the diagram involving $\tilde{\chi}^{ \pm}-\widetilde{v}_{\mu}$ loops displayed in Fig. 2 (left), similarly to the MSSM. This implies that appropriately decreasing the masses of the LH muon-sneutrino $\widetilde{v}_{\mu}$ and charginos $\tilde{\chi}^{ \pm}$is sufficient to lead to a significant enhancement of $a_{\mu}$. In addition, as in the MSSM $a_{\mu}$ increases also with increasing $\tan \beta$. The contribution from the diagram involving $\tilde{\chi}^{0}-\tilde{\mu}$ loops displayed in Fig. 2 (right), is typically smaller than the $\tilde{\chi}^{ \pm}-\widetilde{v}_{\mu}$ one (see e.g. Refs. [82-84]). Nevertheless, we will include in our analysis variations in the masses of neutralinos $\widetilde{\chi}^{0}$ and smuons $\tilde{\mu}$. In this way, $a_{\mu}^{\mu \nu \mathrm{SSM}}$ could have a further increase for low values of these masses.

Summarizing, for reproducing the value of $a_{\mu}$ we are interested in light charginos, neutralinos, LH muon-sneutrino and smuon, and RH smuon, while the other SUSY particles can be decoupled.

Concerning $\tilde{\chi}^{ \pm}$, they mix with the charged leptons in the $\mu \nu \mathrm{SSM}$ but the mixing terms are suppressed, and both type of particles are effectively decoupled. Therefore $\tilde{\chi}^{ \pm}$are of the MSSM type composed of charged winos and higgsinos, thus $m_{\tilde{\chi}^{ \pm}}$are determined by $M_{2}$ and $\mu$. Note that $M_{2}$ is also a crucial parameter for neutrino physics (12), and that $\mu$ is not an independent parameter as shown in Eq. (10).

As discussed above, $\tilde{\chi}^{0}$ and $v_{i R}$ are mixed. As we can see from Eq. (17), a moderate/large negative value of $T_{\kappa}$ is necessary to have heavy pseudoscalar $\mathrm{RH}$ sneutrinos, but this implies that the value of $\kappa$ has to be large enough in order to avoid too light (even tachyonic) scalar RH sneutrinos. As a consequence, large Majorana masses (see Eq. (10)) are generated for RH neutrinos. Therefore, the decoupling of
RH sneutrinos implies also heavy $v_{i R}$, and, in this case, $\widetilde{\chi}^{0}$ are of the MSSM type and composed of neutral EW gauginos and higgsinos. Thus $m_{\tilde{\chi}^{0}}$ are determined by $M_{1}, M_{2}$ and $\mu$, where the $M_{1}$ parameter is also crucial for neutrino physics (12).

Concerning smuon and LH muon-sneutrino masses, we already discussed their masses and showed how they introduce the relevant parameters in Eq. (15) for the analysis of $a_{\mu}$.

From this discussion, we conclude that of the parameters controlling the SUSY contributions to $a_{\mu}$, i.e. $M_{2}, M_{1}, \mu$, $m_{\widetilde{v}_{\mu}}, m_{\tilde{\mu}_{L}}, m_{\widetilde{e}_{2 R}}$ and $\tan \beta$, only

$M_{2}, M_{1}, m_{\widetilde{e}_{2 R}}, \tan \beta$,

are independent in this scenario, and besides $M_{1}$ and $M_{2}$ are also important for neutrino physics, and $\tan \beta$ for Higgs physics.

In our analysis of Sect. 4, we will sample the relevant parameter space of the $\mu \nu \mathrm{SSM}$, which contains the independent parameters determining neutrino, slepton and Higgs physics in Eqs. (12), (15) and (18). Nevertheless, let us point out that the parameters for neutrino physics $Y_{v_{i}}, v_{i L}, M_{1}$ and $M_{2}$ are essentially decoupled from the parameters controlling Higgs physics. Thus, for a suitable choice of $Y_{v_{i}}, v_{i L}, M_{1}$ and $M_{2}$ reproducing neutrino physics, there is still enough freedom to reproduce in addition Higgs data by playing with $\lambda, \kappa, v_{R}, \tan \beta$, etc., as shown in Refs. $[1,67]$. As a consequence, in Sect. 4 we will not need to scan over most of the latter parameters, relaxing our demanding computing task. Given the still large number of independent parameters we have employed the Mul t inest [85] algorithm as optimizer. To compute the spectrum and the observables we have used SARAH [86] to generate a SPheno $[87,88]$ version for the model. In this way, we have evaluated the $a_{\mu}^{\mu \nu \text { SSM }}$ contribution to $a_{\mu}$.

\subsection{Experimental constraints}

The $a_{\mu}$ constraint in Eq. (5) was applied at the $\pm 2 \sigma$ level. All other experimental constraints (except the LHC searches) are taken into account as follows:

- Neutrino observables

We have imposed the results for normal ordering from Ref. [59], selecting points from the scan that lie within 
$\pm 3 \sigma$ of all neutrino observables. On the viable obtained points we have imposed the cosmological upper bound on the sum of the masses of the light active neutrinos given by $\sum m_{v_{i}}<0.12 \mathrm{eV}$ [89].

- Higgs observables

The Higgs sector of the $\mu \nu$ SSM is extended with respect to the (N)MSSM. For constraining the predictions in that sector of the model, we have interfaced HiggsBounds v5.3.2 [90-94] with Multinest, using a conservative $\pm 3 \mathrm{GeV}$ theoretical uncertainty on the SM-like Higgs boson in the $\mu \nu \mathrm{SSM}$ as obtained with SPheno. (As mentioned above, more refined calculations are possible, but would result only in shifts in the colored sector, which does not play a relevant role in our analysis.). Also, in order to address whether a given Higgs scalar of the $\mu \nu \mathrm{SSM}$ is in agreement with the signal observed by ATLAS and CMS, we have interfaced Higgs Signals v2.2.3 [95,96] with Multinest. We require that the $p$-value reported by Higgs Signals be larger than $5 \%$.

- $B$ decays

$b \rightarrow s \gamma$ occurs in the SM at leading order through loop diagrams. We have constrained the effects of new physics on the rate of this process using the average experimental value of $\mathrm{BR}(b \rightarrow s \gamma)=(3.55 \pm 0.24) \times 10^{-4}$ provided in Ref. [97]. Similarly to the previous process, $B_{s} \rightarrow \mu^{+} \mu^{-}$and $B_{d} \rightarrow \mu^{+} \mu^{-}$occur radiatively. We have used the combined results of LHCb and CMS [98], $\operatorname{BR}\left(B_{s} \rightarrow \mu^{+} \mu^{-}\right)=(2.9 \pm 0.7) \times 10^{-9}$ and $\operatorname{BR}\left(B_{d} \rightarrow \mu^{+} \mu^{-}\right)=(3.6 \pm 1.6) \times 10^{-10}$. We put $\pm 3 \sigma$ cuts from $b \rightarrow s \gamma, B_{s} \rightarrow \mu^{+} \mu^{-}$and $B_{d} \rightarrow \mu^{+} \mu^{-}$, as obtained with SPheno. We have also checked that the values obtained are compatible with the $\pm 3 \sigma$ of the recent results from the LHCb collaboration [99].

- $\mu \rightarrow e \gamma$ and $\mu \rightarrow e e e$

We have also included in our analysis the constraints from $\operatorname{BR}(\mu \rightarrow e \gamma)<4.2 \times 10^{-13}[100]$ and $\operatorname{BR}(\mu \rightarrow e e e)<$ $1.0 \times 10^{-12}$ [101], as obtained with SPheno.

- Chargino mass bound

In $R$-parity conserving (RPC) SUSY, the lower bound on the lightest chargino mass of about $94 \mathrm{GeV}$ depends on the spectrum of the model $[3,41]$. Although in the $\mu \nu \mathrm{SSM}$ there is RPV and therefore this constraint does not apply automatically, we have chosen in our analysis a conservative limit of $m_{\tilde{\chi}_{1}^{ \pm}}>92 \mathrm{GeV}$.

In addition, depending on the different masses and orderings of the lightest SUSY particles of the spectra found in our scan, we expect different signals at colliders. Besides, depending on the values of the $\mu \nu$ SSM parameters, the decay of the LSP can be prompt or displaced. Altogether, there is a variety of possible signals arising from the regions of the parameter space analyzed, that we will able to constrain using

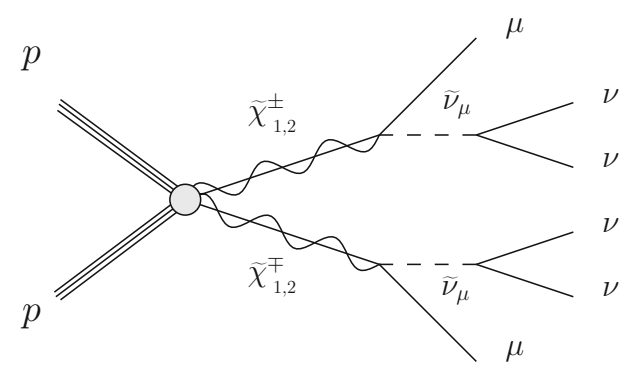

Fig. 3 Production of a chargino pair, each decaying to a LH muonsneutrino, which in turn decays to neutrinos, giving rise to the signal $2 \mu+$ MET

the LHC searches of Refs. [35,38,102]. We will discuss these searches in detail in the next subsection.

\subsection{LHC searches}

The different masses and orderings of the lightest SUSY particles of the spectra found in our analysis of the $\mu \nu \mathrm{SSM}$ parameter space can be classified in four cases:

(i) LH muon-sneutrino $\widetilde{v}_{\mu}$ is the LSP.

When $\widetilde{v}_{\mu}$ is the LSP its main decay channel corresponds to neutrinos [52,65,67], which constitute an invisible signal. Limits on sneutrino LSP from mono-jet and mono-photon searches have been discussed in the context of the $\mu \nu \mathrm{SSM}$ in Refs. [65,67], and they turn out to be ineffective to constrain it. However, the presence of charginos and neutralinos in the spectrum with masses not far above the LSP mass is important for multi-lepton+MET searches. The relevant signals arise from the production of wino/higgsino-like chargino pairs at the LHC, which can give rise to $2 \mu+4 v$ as shown in Fig. 3. These processes produce a signal similar to the one expected from a directly produced pair of smuons decaying as $\tilde{\mu} \rightarrow \mu+\tilde{\chi}^{0}$ in RPC models. Therefore, they can be compared with the limits obtained by the ATLAS collaboration in the search for sleptons in events with two leptons + MET [35].

Other decay modes are possible for the wino-like charginos, in particular chains involving higgsinos when $M_{2}>\mu$. We have also considered the signals produced in events where two higgsino-like neutralinos are directly produced and decay into two smuons plus two muons, giving rise to a final signal with $4 \mu+$ MET. This signal can be compared with the ATLAS search for SUSY in events with four or more leptons [36]. In this scenario, we have also considered the search for events with 2 leptons + MET [35] or 3 leptons + MET [37], in the case where one or two of the muons would remain undetected. As discussed in Ref. [1], all these types of events cannot constrain our parameter space.

There is a small number of points where the LH muonsneutrino is the LSP and the RH smuon is slightly heavier. 


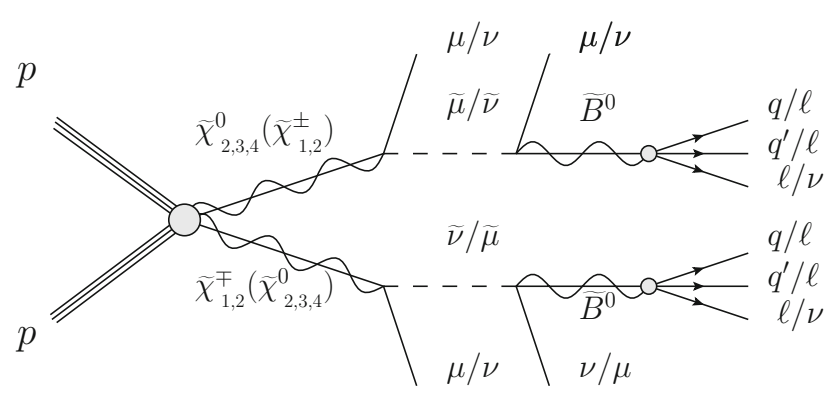

Fig. 4 Production of a chargino pair, chargino-neutralino or a neutralino pair, each decaying to a LH muon-sneutrino or smuon, which in turn decay to a long-lived bino-like neutralino giving rise to a displaced signal

For those points, the events initiated by RH smuon pair can produce a significant number of events including leptons and missing transverse energy, however the energy of the final states is going to be too small to produce constraints.

(ii) Bino-like neutralino $\widetilde{B}^{0}$ is the LSP and $\widetilde{v}_{\mu}$ is the NLSP.

$\widetilde{B}^{0}$ can be the LSP, with $\widetilde{v}_{\mu}$ lighter than wino-like chargino/neutralino and higgsino-like chargino/neutralino and therefore the next-to-LSP (NLSP), i.e. $m_{\widetilde{B}^{0}}<m_{\widetilde{v}_{\mu}}<$ $m_{\widetilde{W}}, \widetilde{H}$, where we denote both $\widetilde{W}^{ \pm}$and $\widetilde{W}^{0}\left(\widetilde{H}^{ \pm}\right.$and $\left.\widetilde{H}^{0}\right)$ generically as $\widetilde{W}(\widetilde{H})$. Then, the proton-proton collisions produce a pair chargino-chargino, chargino-neutralino or neutralino- neutralino as shown in Fig. 4. The charginos and neutralinos will rapidly decay to sneutrinos/smuons and muons/neutrinos, with the former subsequently decaying to neutrinos/muons plus binos. When $m_{\widetilde{B}^{0}} \lesssim m_{W}$ (with $m_{W}$ denoting the mass of the $W$ boson), $\widetilde{B}^{0}$ decay is suppressed in comparison with the one of $\widetilde{v}_{\mu}$ LSP. This originates in the kinematical suppression associated with the three-body nature of the $\widetilde{B}^{0}$ decay. For this reason, it is natural that the $\widetilde{B}^{0}$ proper decay length is an order of magnitude larger than the one of $\widetilde{v}_{\mu}$, being therefore of the order of ten centimeters. The points of the parameter space where the LSP decays with a proper decay distance larger than $1 \mathrm{~mm}$ can be constrained applying the limits on long-lived particles (LLPs) obtained by the ATLAS $8 \mathrm{TeV}$ search [38]. Let us point out that we have used this search instead of the more recent $13 \mathrm{TeV}$ one [39], because it tests all the possible decay channels of $\widetilde{B}^{0}$ while the latter focuses exclusively on leptonic displaced vertices. The conclusion is that no points of our parameter space can be excluded by the most recent analysis.

When $m_{\widetilde{B}^{0}} \gtrsim 130 \mathrm{GeV}$ the two-body nature of its decay implies that its decay length, $c \tau$, becomes smaller than $1 \mathrm{~mm}$. In that case, following the strategy discussed in Ref. [1] we can apply ATLAS searches [35] based on the promptly produced leptons in the decay of the heavier chargino-neutralino.

We have also considered here, and in (iii) (see below), whether the case of the direct production of a smuon pair, smuon-sneutrino or a sneutrino pair, with the smuon/sneutrino

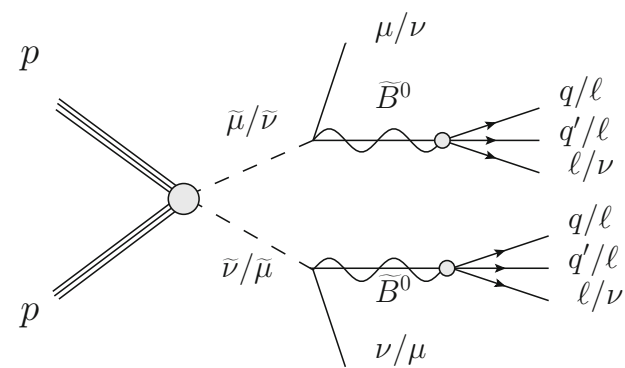

Fig. 5 Production of a smuon pair, smuon-sneutrino or a sneutrino pair, each decaying to a long-lived bino-like neutralino giving rise to a displaced signal

decaying into a muon/neutrino and a long lived $\widetilde{B}^{0}$, could produce a significant signal, as shown in Fig. 5. The number of events predicted in this way is added to the events produced as shown in Fig. 4, and the result is that the combination of both signals excludes some points of the parameter space which are not excluded analyzing each signal separately.

(iii) $\widetilde{B}^{0}$ is the LSP and $\widetilde{W}$ or $\widetilde{H}$ are co-NLSPs.

The situation in this case with $m_{\widetilde{B}^{0}}<m_{\widetilde{W}, \widetilde{H}}<m_{\widetilde{v}_{\mu}}$ is similar to the one presented in (ii), with the difference in the particles produced in the intermediate decay, as shown in Fig. 6. While before, this corresponded in most cases to muons, now the intermediate decay will mainly produce hadrons. The LHC constraints are applied in an analogous way, depending also on the value of the proper decay length, larger or smaller than $1 \mathrm{~mm}$.

(iv) RH smuon $\tilde{\mu}_{R}$ is the LSP.

When $\tilde{\mu}_{R}$ is the LSP the limits from LEP [103-108] exclude masses smaller than $96.3 \mathrm{GeV}$ for any value of its lifetime. For the rest of the points with larger values of the mass we study the following process at the LHC: once produced $\tilde{\mu}_{R}$ decays to a muon and a neutrino mediated by the small bino composition of the latter in the mass basis, as shown in Fig. 7. When $\tilde{\mu}_{R}$ decay is sufficiently suppressed to yield a proper decay length larger than $3 \mathrm{~mm}$, it is constrained by the search for displaced leptons at ATLAS [102]. This search is able to constrain $\tilde{\mu}_{R}$ up to a mass of $500 \mathrm{GeV}$. We impose the limits that are extracted from Fig. 11d of the auxiliary material of Ref. [109]. On the contrary, if the decay of $\widetilde{\mu}_{R}$ is fast enough to be considered prompt, it can be constrained using the search for EW production of sleptons decaying into final states with two leptons and missing transverse momentum [35]. This search imposes a lower limit of $450 \mathrm{GeV}$ on the mass of $\tilde{\mu}_{R}$. Note that if the decay length of $\widetilde{\mu}_{R}$ is too long to be analyzed as prompt, but shorter than $3 \mathrm{~mm}$, the LHC searches are not able to put limits on its mass.

It is worth noting here that when $\widetilde{\mu}_{R}$ is heavier than other SUSY particles, there can be three situations of interest. In one of them, $\widetilde{B}^{0}$ is the LSP and $\widetilde{\mu}_{R}$ is the NLSP. Then, the decay of $\widetilde{\mu}_{R}$ will produce a signal similar to the one shown in 


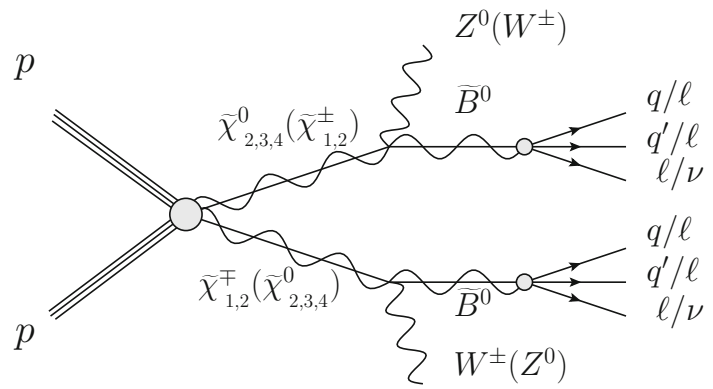

Fig. 6 Production of a chargino pair, chargino-neutralino or a neutralino pair, each decaying to a long-lived bino-like neutralino giving rise to a displaced signal

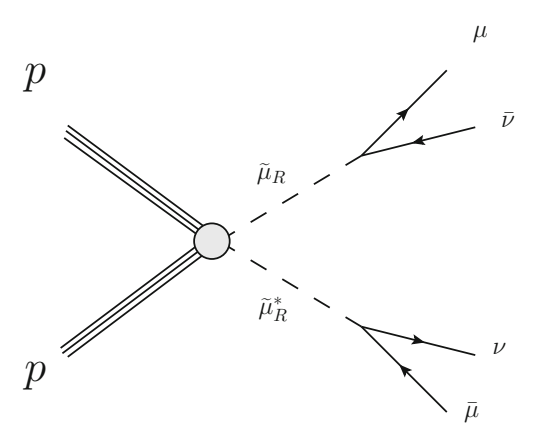

Fig. 7 Production of RH smuon pair, each decaying to a RH muon and neutrino, giving rise to the signal $2 \mu+$ MET

Fig. 4, but without the presence of neutrinos coming from the first step of the decay. This signal can be treated therefore in a similar way, now considering the production cross-section of a $\widetilde{\mu}_{R}$. Another situation occurs when $\widetilde{v}_{\mu}$ is the LSP and $\widetilde{\mu}_{R}$ the NLSP. The dominant decay chain in this case is $\tilde{\mu}_{R} \rightarrow$ $W(\rightarrow l+v)+\widetilde{v}_{\mu}(\rightarrow v v)$, which is similar to the one analyzed in Ref. [35] (see their Fig. 1a). Finally, we can have $\widetilde{v}_{\mu}$ as the LSP, $\widetilde{B}^{0}$ as the NLSP, and $\widetilde{\mu}_{R}$ slightly heavier than both of them. In this case, the dominant $\widetilde{\mu}_{R}$ decay chain is $\widetilde{\mu}_{R} \rightarrow$ $\widetilde{B}^{0}+\mu \rightarrow v+\widetilde{v}_{\mu}(\rightarrow v v)+\mu$, and this signal is similar to the one analyzed in Ref. [35] (see their Fig. 1b). We conclude that the limits for a $\tilde{\mu}_{R}$ heavier than the LSP, imposed as described above, do not exclude any additional point.

\section{Parameter analysis}

We describe in this section the methodology that we have employed to search for points of the parameter space of the $\mu \nu \mathrm{SSM}$ that are compatible with the given experimental data. To carry out this analysis we will follow the same strategy as in Ref. [1]. This consists of combining a scan of the relevant parameters to obtain acceptable points, with a subsequent intelligent search to increase the number of points obtained. It is worth noting here that we will not perform any sta- tistical interpretation of the set of points obtained, i.e. the Multinest algorithm is just used to obtain viable points.

The Higgsino mass parameter $\mu$ is important in our analysis, since it is one of the parameters controlling the SUSY contributions to $a_{\mu}$ as discussed in Sect. 2.1. Besides, since higgsinos have a mass of order $\mu$, its value has also important implications for the analysis of the LHC constraints discussed in Sect. 2.3. Thus, in order to have an idea of how $a_{\mu}^{\mu \nu S S M}$ varies with $\mu$, it is interesting to use two representative values of this parameter in our computation. In particular, we will use a moderate value $\mu \approx 380 \mathrm{GeV}$, and a large value $\mu \approx 800 \mathrm{GeV}$.

\section{(1) Moderate $\mu \approx 380 \mathrm{GeV}$.}

We will perform as a first step a scan, using the fewest possible parameters in order to relax our demanding computing task. As discussed in Sect. 2.1, the most crucial parameters for neutrino physics (12) are basically decoupled from those controlling Higgs physics (18). Thus, for a suitable scan of $Y_{v_{i}}, v_{i}, M_{1}$ and $M_{2}$ reproducing neutrino physics, there is still enough freedom to reproduce in addition Higgs data by playing with concrete values of $\lambda, \kappa, \tan \beta, v_{R}$, etc. For $\tan \beta$ we will consider a narrow range of possible values compatible with Higgs physics. On the other hand, LH sneutrino masses $m_{\widetilde{v_{i}}}$, introduce in addition the parameters $T_{v_{i}}$ (see Eq. (14)). In particular, $T_{\nu_{2}}$ is the most relevant one for our discussion of a low $m_{\widetilde{v}_{\mu}}$, and we will scan it in an appropriate range of small values. Since the LH sneutrinos of the other two generations can be heavier, we can fix $T_{\nu_{1,3}}$ to a larger value.

Summarizing, in our first step we will perform a scan over the 9 parameters $Y_{v_{i}}, v_{i}, T_{v_{2}}, \tan \beta, M_{2}$, as shown in Table 1. Concerning $M_{1}$, we will assume for the EW gauginos $M_{1}=M_{2} / 2$. This relation is inspired by GUTs, where the low-energy result $M_{2}=\left(\alpha_{2} / \alpha_{1}\right) M_{1} \simeq 2 M_{1}$ is obtained, with $g_{2}=g$ and $g_{1}=\sqrt{5 / 3} g^{\prime}$. The ranges of $v_{i}$ and $Y_{v_{i}}$ are natural in the context of the EW scale seesaw of the $\mu \nu \operatorname{SSM}[1,67]$. The range of $T_{\nu_{2}}$ is chosen to have light $\widetilde{v}_{\mu}$ below about $800 \mathrm{GeV}$. This is a reasonable upper bound to be able to have sizable SUSY contributions to $a_{\mu}$. In the supergravity framework where $T_{\nu_{2}}=A_{\nu_{2}} Y_{\nu_{2}}$, this implies $-A_{\nu_{2}} \in\left(1,4 \times 10^{4}\right) \mathrm{GeV}$.

Other benchmark parameters relevant for Higgs physics are fixed to appropriate values, as shown in Table 1 . We choose a small/moderate value for $\lambda \approx 0.1$, thus we are in a similar situation as in the MSSM and moderate/large values of $\tan \beta,\left|T_{u_{3}}\right|$, and scalar top masses are necessary to obtain through loop effects the correct SM-like Higgs mass [7780]. To avoid the chargino mass bound of RPC SUSY, this value of $\lambda$ forces us to choose a moderate/large value of $v_{R}$ to obtain a large enough value of $\mu$ (see Eq. (10)). We choose $v_{R}=1750 \mathrm{GeV}$ giving rise to $\mu=378.7 \mathrm{GeV}$. Thus in our scan the value of $\mu$ is fixed, as discussed above. Another parameter controlling the SUSY contributions to $a_{\mu}$ is $m_{\widetilde{e}_{2 R}}$ 
Table 1 Low-energy values of the input parameters in the scan discussed in Case (1) of the text. Soft SUSY-breaking parameters and vevs are given in $\mathrm{GeV}$

\begin{tabular}{ll}
\hline Parameters & Scan \\
\hline $\tan \beta$ & $(10,16)$ \\
$Y_{v_{i}}$ & $\left(10^{-8}, 10^{-6}\right)$ \\
$v_{i}$ & $\left(10^{-6}, 10^{-3}\right)$ \\
$-T_{\nu_{2}}$ & $\left(10^{-6}, 4 \times 10^{-4}\right)$ \\
$M_{2}$ & $(150,1000)$ \\
$\lambda$ & 0.102 \\
$\kappa$ & 0.4 \\
$v_{R}$ & 1750 \\
$T_{\lambda}$ & 340 \\
$-T_{\kappa}$ & 390 \\
$-T_{u_{3}}$ & 4140 \\
$m_{\widetilde{Q}_{3 L}}$ & 2950 \\
$m_{\widetilde{u}_{3 R}}$ & 1140 \\
$m_{\widetilde{e}_{2 R}}$ & 1000 \\
$m_{\widetilde{e}_{1,3 R}}$ & 1000 \\
$m_{\widetilde{Q}_{1,2 L}}, m_{\widetilde{u}_{1,2 R}}, m_{\tilde{d}_{1,2,3 R}}$ & 1000 \\
$M_{3}$ & 2700 \\
$T_{u_{1,2}}$ & 0 \\
$T_{d_{1,2}}, T_{d_{3}}$ & 0,100 \\
$T_{e_{1,2}}, T_{e_{3}}$ & 0,40 \\
$-T_{\nu_{1,3}}$ & $10^{-3}$ \\
\hline &
\end{tabular}

(see Eq. (19)), and we fix it in the scan to the large value $m_{\widetilde{e}_{2 R}}=1000 \mathrm{GeV}$. The parameters $\kappa$ and $T_{\kappa}$ are crucial to determine the mass scale of the RH sneutrinos. We choose $T_{\kappa}=-390 \mathrm{GeV}$ to have heavy pseudoscalar RH sneutrinos (of about $1190 \mathrm{GeV}$ ), and therefore the value of $\kappa$ has to be large enough in order to avoid too light (even tachyonic) scalar RH sneutrinos. Choosing $\kappa=0.4$, we get masses for the latter of about 700-755 GeV. This value of $\kappa$ (and the one chosen above for $v_{R}$ ) also implies that the Majorana mass is fixed to $\mathcal{M}=989.9 \mathrm{GeV}$. The parameter $T_{\lambda}$ is relevant to obtain the correct values of the off-diagonal terms of the mass matrix mixing the RH sneutrinos with Higgses, and we choose for its value $340 \mathrm{GeV}$. The values of the parameters shown below the soft SUSY-breaking RH smuon mass $m_{\widetilde{e}_{2 R}}$ in Table 1 concern slepton, squark and gluino masses, as well as quark and lepton trilinear parameters, which are not specially relevant for our analysis of $a_{\mu}$. The values chosen for $T_{\nu_{1,3}}$ are larger that the one of $T_{\nu_{2}}$, but they are natural within the supergravity framework where $T_{\nu_{13}}=A_{\nu_{1,3}} Y_{\nu_{1,3}}$, since then larger values of the Yukawa couplings are required for similar values of $A_{v_{i}}$. This allows us to reproduce the normal ordering of neutrino masses with $Y_{\nu_{2}}<Y_{\nu_{1}}<Y_{\nu_{3}}[1,67]$ (see the discussion in Eq. (13)). In a similar way, the val- ues of $T_{d_{3}}$ and $T_{e_{3}}$ have been chosen taking into account the corresponding Yukawa couplings.

The second step of our analysis of the $\mu \nu$ SSM parameter space, consists of using suitable points from the previous scan, varying appropriately some of their associated parameters in order to explore other regions, where the new points still fulfill the experimental constraints. In particular, note that in fact neutrino physics depends mainly on the parameter $M$ defined in Eq. (11). This implies that for a given value of $M$ reproducing the correct neutrino (and Higgs) physics, one can get different pairs of values of $M_{1}$ and $M_{2}$ with the same good properties, without essentially modifying the values of the other parameters. This allows us to break the GUT-inspired relation $M_{2}=2 M_{1}$, to explore other interesting regions of the parameter space. On the other hand, given the value of $m_{\widetilde{v}_{\mu}}$ obtained for each point, one can find more suitable points but with a different mass just varying $T_{\nu_{2}}$, since this parameter does not affect the neutrino (and Higgs) physics. In addition, one can also lower the soft RH smuon mass, which leads to an enhancement of $a_{\mu}^{\mu \nu \mathrm{SSM}}$ as discussed in Sect. 2.1.

\section{(2) Large $\mu \approx 800 \mathrm{GeV}$.}

Although the value of $\mu$ used above is reasonable, many other values reproducing the correct Higgs physics can be obtained [79], and in particular larger ones. Thus we have also studied points with $\lambda=0.126$, similar to the value above, but with a larger value for the vev $v_{R}=3000 \mathrm{GeV}$ giving rise to $\mu=801.9 \mathrm{GeV}$. Other benchmark parameters relevant for Higgs physics have to be modified, such as $\kappa=0.36$ yielding $\mathcal{M} \approx 1527.4 \mathrm{GeV},-T_{\kappa}=150 \mathrm{GeV}$, $T_{\lambda}=1000 \mathrm{GeV},-T_{u_{3}}=4375 \mathrm{GeV}, m_{\widetilde{Q}_{3 L}, \widetilde{u}_{3 R}}=2500 \mathrm{GeV}$ and $M_{3}=3500 \mathrm{GeV}$. For the other squarks masses and the slepton masses we use $m_{\widetilde{Q}_{1,2 L}}, m_{\widetilde{u}_{1,2 R}}, m_{\widetilde{d}_{1,2,3 R}}, m_{\widetilde{e}_{1,2,3 R}}=$ $1500 \mathrm{GeV}$. We have also modified the range of $\tan \beta$ with respect to the one in Table 1 , using $\tan \beta \in(25,35)$. Concerning the LH muon-sneutrino mass, we have slightly increased the upper limit of $-T_{\nu_{2}}$ up to $4.4 \times 10^{-4} \mathrm{GeV}$, and to obtain slightly smaller chargino masses we have decreased the lower limit of $M_{2}$ up to $100 \mathrm{GeV}$. The rest of the parameters are taken as in Table 1.

Unlike the previous Case (1), instead of starting with a scan we simplify our analysis choosing several benchmark points with correct Higgs and neutrino physics, and apply to them the variation of relevant parameters discussed in the second step above. In particular, for a given $M$ we vary $M_{1}$ and $M_{2}$, and for a given $m_{\widetilde{v}_{\mu}}$ we vary $T_{\nu_{2}}$. Obviously, more acceptable points could have been obtained with a scan, but as we will see in the next section the ones obtained are sufficient to have a good idea of the interesting regions of the $\mu \nu \mathrm{SSM}$ parameter space when $\mu$ is large. 


\section{Results}

We present here the results obtained following the strategy discussed in the previous section. In particular, we use the new combined $(g-2)_{\mu}$ result at the $\pm 2 \sigma$ level to obtain upper (and lower) bounds on the EW SUSY masses. We denote the points in different regions of the parameter space, and surviving certain constraints, with different colors and symbols as shown in Figs. 8 and 9. Concerning the colors, they denote the regions where the points are located in the $\mu \nu \mathrm{SSM}$ parameter space, as well as their agreement with the new world average of $a_{\mu}^{\exp }$ specifying if they are in the $1 \sigma$ or $2 \sigma$ regions of $\Delta a_{\mu}$ in Eq. (5):

- Dark-green $(1 \sigma)$ and dark-blue $(2 \sigma)$ points correspond to Case (1) of the previous section with $m_{\widetilde{e}_{2 R}}=1000 \mathrm{GeV}$.

- Light-green $(1 \sigma)$ and light-blue $(2 \sigma)$ points are obtained from the dark-green ones but using $m_{\widetilde{e}_{2 R}}=100,150$, 200, 300, $500 \mathrm{GeV}$.

- Orange $(2 \sigma)$ points correspond to Case (2) of the previous section with $m_{\widetilde{e}_{2 R}}=1500 \mathrm{GeV}$.

In addition, each point surviving LHC searches can be classified in the four categories discussed in Sect. 2.3, depending on the different relevant signals that it produces at colliders. We use the following symbols:

(i) Dots (o) are points with $\widetilde{v}_{\mu}$ LSP.

(ii) Crosses (+) are points with $\widetilde{B}^{0}$ LSP and the hierarchy $m_{\widetilde{B}^{0}}<m_{\widetilde{v}_{\mu}}<m_{\widetilde{W}, \widetilde{H}}$.

(iii) Triangles $(\triangle)$ are points with $\widetilde{B}^{0}$ LSP and the hierarchy $m_{\widetilde{B}^{0}}<m_{\widetilde{W}, \tilde{H}}<m_{\widetilde{v}_{\mu}}$.

(iv) Stars ( $\star$ ) are points with $\tilde{\mu}_{R}$ LSP.

We show in Fig. 8 the preferred parameter ranges in the $M_{2}-m_{\tilde{v}_{\mu}}$ plane. As one can see, significant regions of the parameter space of the $\mu \nu \mathrm{SSM}$ can be found being compatible with the experimental results from neutrino and Higgs measurements, as well as with flavor observables, and fulfilling the constraints from the LHC Run 1 and 2, as discussed in Sects. 2.2 and 2.3. In particular, they are in agreement with the new world average for $a_{\mu}^{\exp }$. LH muon-sneutrino masses are found in the range $120 \mathrm{GeV} \lesssim m_{\widetilde{v}_{\mu}} \lesssim 540 \mathrm{GeV}$, and $202 \mathrm{GeV} \lesssim M_{2} \lesssim 560 \mathrm{GeV}$. These values of $M_{2}$ correspond to wino-like chargino/neutralino masses in the range $200 \mathrm{GeV} \lesssim m_{\widetilde{W}} \lesssim 597 \mathrm{GeV}$. The corresponding values of $M_{1}$ compatible with neutrino physics are smaller than $M_{2}$, and they are in the range $117 \mathrm{GeV} \lesssim M_{1} \lesssim$ $378 \mathrm{GeV}$. The range of bino-like neutralino masses is therefore $114 \mathrm{GeV} \lesssim m_{\widetilde{B}^{0}} \lesssim 370 \mathrm{GeV}$. The values used for $\mu \approx 380 \mathrm{GeV}, 800 \mathrm{GeV}$, correspond to higgsino-like chargino/neutralino masses in the range $333 \mathrm{GeV} \lesssim m_{\widetilde{H}} \lesssim$ $878 \mathrm{GeV}$.

In Fig. 9, we show as complementary information $a_{\mu}^{\mu \nu \text { SSM }}$ versus $M_{2}$. As we can see from both figures, the smaller $M_{2}\left(m_{\widetilde{v}_{\mu}}\right)$ is, the larger $a_{\mu}^{\mu \nu S S M}$ becomes as expected. One can also see that the orange points with a large value of $\mu$ $(=801.9 \mathrm{GeV})$ do not enter in the $1 \sigma$ region of $\Delta a_{\mu}$, unlike the dark- and light-green points with $\mu=378.7 \mathrm{GeV}$. As mentioned above, we have also analyzed how the $a_{\mu}^{\mu \nu \text { SSM }}$ values obtained can be enhanced lowering the value of $m_{\tilde{\mu}_{R}}$. In particular, we have focused on the dark-green points of the figures, which are in the $1 \sigma$ region of $\Delta a_{\mu}$, using instead of $m_{\tilde{e}_{2 R}}=1000 \mathrm{GeV}$ lower values. These points are shown in light green and light blue in Figs. 8 and 9. As one can see in the two figures, many light-green and light-blue points with an increased neutralino-smuon contribution are found with larger values for $a_{\mu}^{\mu \nu \mathrm{SSM}}$. The maximum value of $a_{\mu}^{\mu \nu \mathrm{SSM}}$ can be as large as about $36 \times 10^{-10}$ for $m_{\tilde{e}_{2 R}}=150 \mathrm{GeV}$. This is to be compared with the maximum value of about $29 \times 10^{-10}$ obtained with $m_{\tilde{e}_{2 R}}=1000 \mathrm{GeV}$ for the dark-green points.

An interesting result of our analysis is that although LHC Runs 1 and 2 are important to constrain the $\mu \nu$ SSM scenario, it is not in fact difficult to find regions where many points are viable as well as compatible with $\Delta a_{\mu}$, as shown in the figures. For example, a significant number of points fulfilling the experimental constrains discussed in Sect. 2.2 turn out to be forbidden by the LHC constraints discussed in Sect. 2.3. This is the case for many points with $\widetilde{B}^{0}$ as the LSP, denoted by crosses and triangles. They are excluded when the GUTinspired relation $M_{2}=2 M_{1}$ is used. This happens for decay lengths larger as well as smaller than $1 \mathrm{~mm}$, applying the displaced and prompt LHC constraints discussed in items (ii) and (iii) of Sect. 2.3. Nevertheless, the situation changes a lot allowing $M_{2} \neq 2 M_{1}$, when many of these points turn out to be unconstrained by LHC searches. All of this kind of points in Figs. 8 and 9 have $117 \mathrm{GeV} \lesssim M_{1} \lesssim 285 \mathrm{GeV}$ corresponding to a proper decay length of $\widetilde{B}^{0}$ LSP in the range $0.1 \mathrm{~mm} \lesssim c \tau_{\widetilde{B}^{0}} \lesssim 1.25 \mathrm{~mm}$, as shown in Fig. 10 .

Similarly, the light-green and light-blue stars corresponding to points with $\tilde{\mu}_{R}$ as the LSP with masses $106 \mathrm{GeV}$ $\lesssim m_{\tilde{\mu}_{R}} \lesssim 190 \mathrm{GeV}$, have a decay length in the range $1.5 \mathrm{~mm}$ $\lesssim c \tau_{\tilde{\mu}_{R}} \lesssim 3 \mathrm{~mm}$, avoiding in this way the LEP and LHC constraints discussed in item (iv) of Sect. 2.3.

Furthermore, more $\widetilde{v}_{\mu}$ LSP-like points are allowed when a larger set of $\widetilde{v}_{\mu}$ masses are explored varying $T_{\nu_{2}}$ for given values of the rest of parameters.

It is worth noting here that a significant number of $\widetilde{v}_{\mu}$ LSP-like points are forbidden because of the limits imposed on the higgsino-like chargino pair production. This can be avoided when the presence of binos with masses between the charginos and the LSP opens new decay channels. In this situation, the signal is divided into different channels that individually don't exceed the experimental limits. These 
Fig. $8 m_{\widetilde{v}_{\mu}}$ versus $M_{2}$ for points in the parameter space of the $\mu \nu \mathrm{SSM}$ in agreement with the experimental constraints

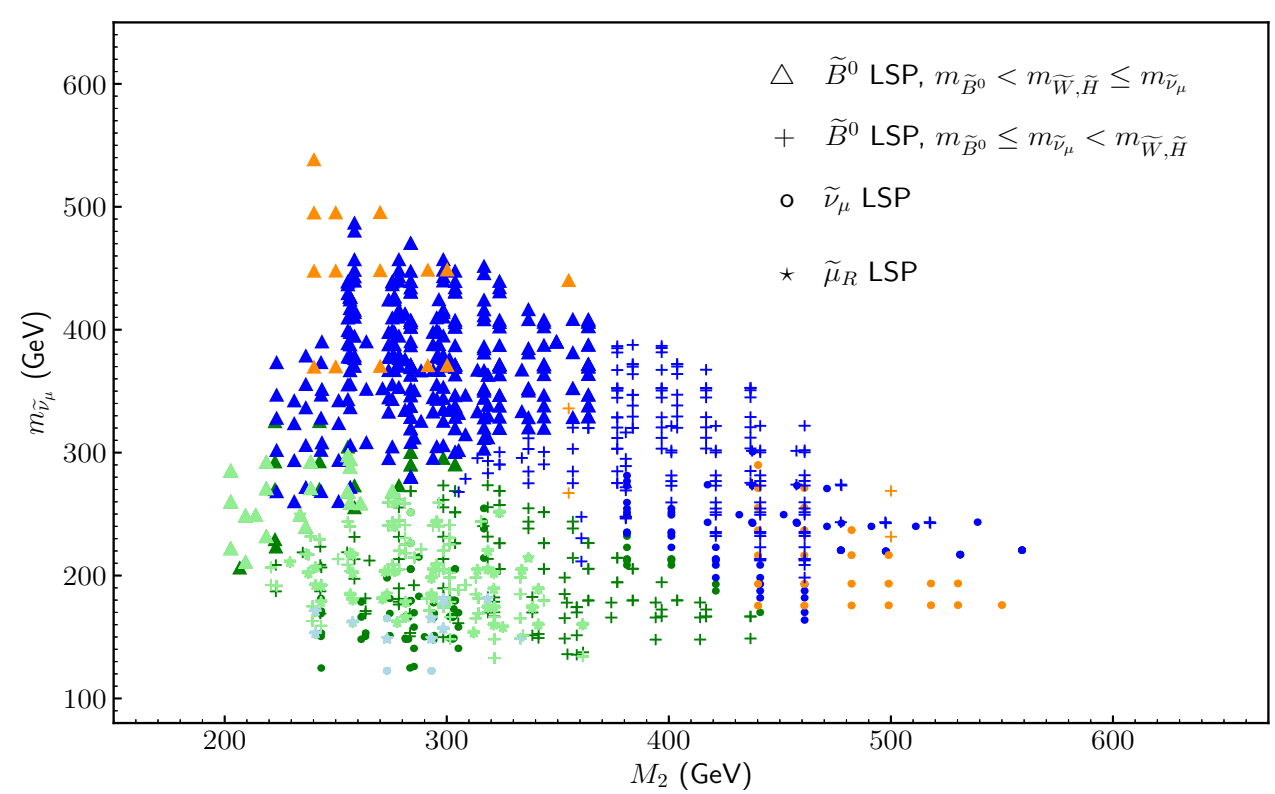

points are shown in Figs. 8 and 9 with dots, and light- and dark-green colors Another way to avoid it is the following. Forbidding dots can occur when $M_{2}>\mu$ and therefore the higgsino is lighter than the wino. Since in our scan we fixed $\mu \approx 380 \mathrm{GeV}$, points with $M_{2} \gtrsim 380 \mathrm{GeV}$ have this hierarchy of masses. Nevertheless, using a larger value of $\mu \approx 800 \mathrm{GeV}$ allows events initiated by higgsinos to pass the selection cuts. These points are shown in Figs. 8 and 9 with dots, and orange colors.

Concerning the above presented analysis the following should be kept in mind. The values of the free parameters found in agreement with the new $a_{\mu}$ data can be considered as a subset of all the solutions that could be obtained if a general scan of the parameter space of the model was carried out. We could have obtained straightforwardly other values for $\tan \beta$, $Y_{v_{i j}}, \lambda, \kappa, v_{i}, v_{R}$, etc., fulfilling all experimental constraints. Nevertheless, we do not expect significant modifications with respect to the viable intervals of the values of $m_{\widetilde{v}_{\mu}}$ and $M_{2}$ shown in Fig. 8, since they correspond to the sensible regions of these parameters which can give rise to a large enough $a_{\mu}^{\mu \nu \mathrm{SSM}}$.

\section{Implications for future collider searches}

As we have seen in the previous section, the parameter points compatible with the new world average for $a_{\mu}$ predict light sleptons and/or gauginos, which can be the prime target for the future (HL-)LHC experiments. Generically speaking, such light EW SUSY particles have already been stringently restricted by the existing LHC results, especially by the multilepton + MET searches [35-37]. Nevertheless, the parameter points shown above in the $1 \sigma$ region of $\Delta a_{\mu}$ evade these limits thanks to (a) metastability of the LSP such as points with triangles, crosses and stars, or (b) close mass spectrum such as points with dots. The importance of mass degeneracy for some of the points may be inferred from the results given in Ref. [1], where both the GUT-inspired relation, $M_{2}=2 M_{1}$, and $M_{2} \neq 2 M_{1}$ cases were analyzed; for $M_{2}=2 M_{1}$, most of the parameter points that induce a sizable value of $a_{\mu}^{\mu \nu \mathrm{SSM}}$ have already been excluded by the LHC experiments, while we can find many allowed points with $M_{2} \neq 2 M_{1}$. A part of the allowed points in Figs. 8 and 9 will be probed in the future multi-lepton + MET searches at the (HL-)LHC, and the rest of them may be explored at a larger hadron collider such as a $100 \mathrm{TeV}$ collider. For the prospects of the electroweak gaugino/slepton searches at the HL-LHC (a $100 \mathrm{TeV}$ collider), see Refs. [110,111] (Refs. [112-114]).

Sleptons and gauginos are more efficiently probed at lepton colliders, such as the ILC [115-117] and the CLIC $[116,118]$, through the pair production of these particles. For the previous studies on the role of lepton colliders in testing the SUSY explanation for $\Delta a_{\mu}$ in the MSSM, see Refs. [48,49,119]. Here usually masses up to the kinematic limit, i.e. $m \lesssim \sqrt{s} / 2$ for pair production, can be probed, see Ref. [120]. This covers compressed spectra as well as possibly meta-stable particles. As obtained from Fig. 8, in the present scenario, the masses of the EW SUSY particles are predicted to be $\gtrsim 114 \mathrm{GeV}$ and only a limited parameter space is accessible to the ILC-250. Nevertheless, most of the $1-\sigma$ points can be probed with a higher energy, such as the ILC-500, CLIC Stage $1(350 / 380 \mathrm{GeV})$, or FCC-ee, and the rest of the points shown in Fig. 8 may be covered at the 1-TeV ILC and CLIC Stage 2 (1.5 TeV). In addition to these electron-positron colliders, a muon collider [121$123]$ is also useful to discover new physics contributing to $\Delta a_{\mu}$ [124-127], since the colliding muons directly couple to it. At a muon collider, not only the EW charged states - 
Fig. 9 The same as in Fig. 8, but showing $a_{\mu}^{\mu \nu \text { SSM }}$ versus $M_{2}$

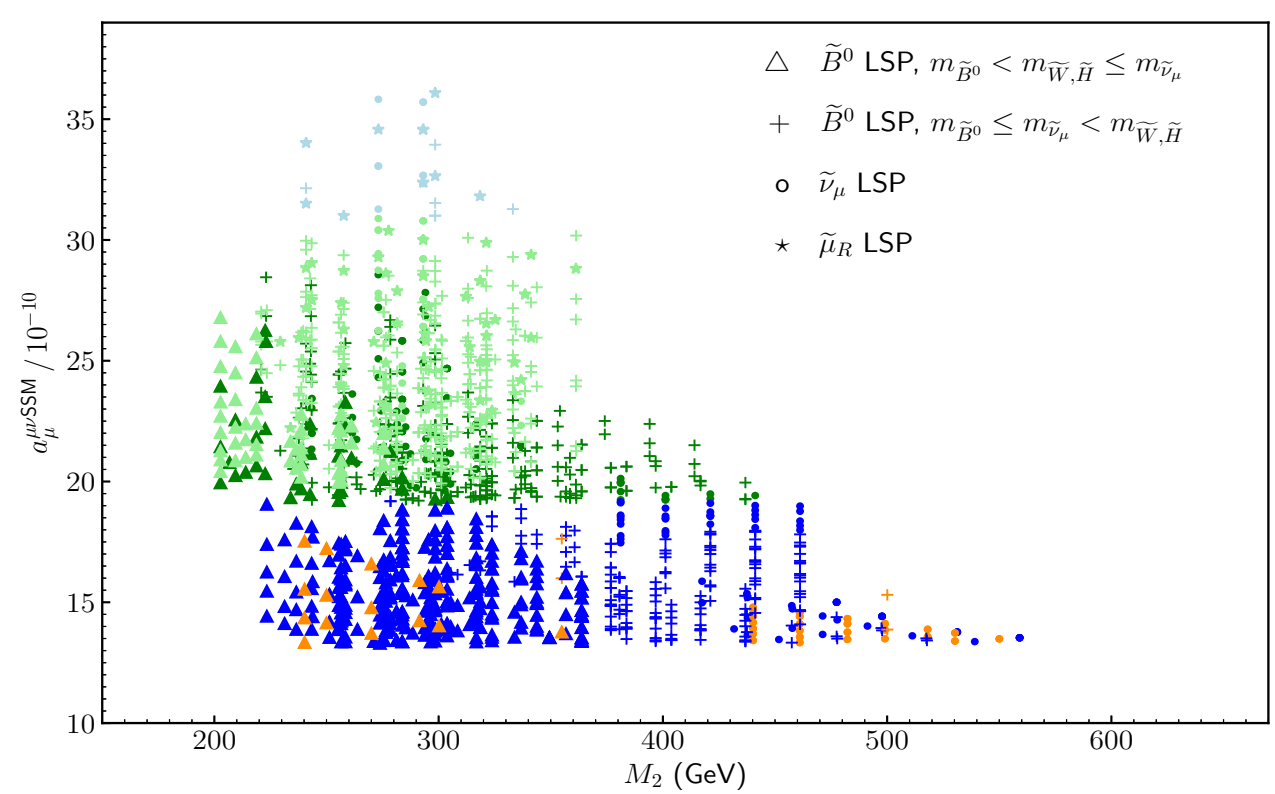

smuons, muon sneutrinos, winos, and higgsinos [128] - but also binos can directly be produced through the $t$-channel smuon exchange. This direct bino production process is useful to determine the mass and lifetime of the bino in the case of the bino LSP, which then allows us to test the characteristic prediction for their correlation, as seen in Fig. 10. We can also carry out a similar analysis for the $\tilde{\mu}_{R}$ LSP. A detailed study on the implications of future lepton colliders on the $\mu \nu \mathrm{SSM}$ will be performed on another occasion.

In addition to the direct searches discussed above, we may probe our scenario in a more indirect manner. For example, the presence of light particles that couple to the Higgs bosons generically deviates the couplings of the SM-like Higgs boson from the SM prediction, and thus we can probe such signature through the precision measurements of the Higgs couplings. However, for this work we chose the parameters such that to obtain a SM-like Higgs rather similar to the SM one, producing a deviation in the Higgs coupling fairly small; for instance, the change in the Higgs-photon (Higgs-muon) coupling is found to be $\lesssim 0.8 \%(0.2 \%)$, which is (far) below the sensitivity of the future precision Higgs measurements [117]. It is, therefore, important to pursue an option for a high-energy future collider that is capable of directly producing the EW particles with a mass of $\gtrsim 114 \mathrm{GeV}$.

Light sleptons and/or gauginos are also predicted in the parameter region of the MSSM where the new world average for $a_{\mu}$ can be explained (for recent studies that discuss the SUSY explanation for $\Delta a_{\mu}$ in the MSSM based on the BNL result, see, for instance, Refs. [48,49,84, 129142]). Thus, a discovery of such an electroweakly charged state by itself cannot distinguish our scenario from the MSSM explanation. We note, however, that in the case of the MSSM, the parameter regions in which sleptons are lighter than charginos are less favored (although not totally excluded, see Refs. [48,49]) by the LHC Run 2 results [84], while such a mass spectrum is still relatively unconstrained in the $\mu \nu \mathrm{SSM}$. Furthermore, in the $\mu \nu \mathrm{SSM}$, sleptons can even be lighter than bino thanks to the $R$-parity violation, which is not allowed in the MSSM. We, therefore, envision that the determination of the mass spectrum of sleptons and gauginos may allow us to discriminate the $\mu \nu \mathrm{SSM}$ from the MSSM. On the other hand, the RH sneutrinos as well as the LH sneutrinos from the first and third generation were chosen to be heavy and thus do not play a role here.

Displaced-vertex searches in the LHC Run 3 or the HLLHC offer another promising way to cover large parts of the favored parameter space, since the presence of a metastable LSP is a characteristic prediction in the $\mu \nu \mathrm{SSM}$. As we discussed above, the parameter points corresponding to the cross and triangle symbols in Figs. 8 and 9 predict a metastable bino, whose decay can be observed as a displaced decay vertex. The points shown in these figures avoid the current bound from the displaced vertex search [38] since the decay length of the bino LSP is rather short, $\lesssim 1.25 \mathrm{~mm}$, as seen in Fig. 10. We note, however, that it is possible to improve the sensitivity of the displaced-vertex searches for a sub-millimeter decay length; given the extremely low background in these searches, we can safely relax the requirements on the impact parameter of lepton tracks and the reconstructed position of displaced vertices, as discussed in Ref. [65]. The decay length of the bino-like neutralino LSP in our model is predicted to be larger than $0.1 \mathrm{~mm}$ [1] (see Fig. 10), which is sufficiently larger than the resolution of the transverse impact parameter, $\sigma\left(d_{0}\right)$ (for instance, $\sigma\left(d_{0}\right) \simeq 0.03 \mathrm{~mm}$ for $p_{T} \gtrsim 10 \mathrm{GeV}$ [143]). For the param- 
Fig. 10 Proper decay length of the bino LSP $c \tau_{\widetilde{B}^{0}}$ versus $M_{1}$

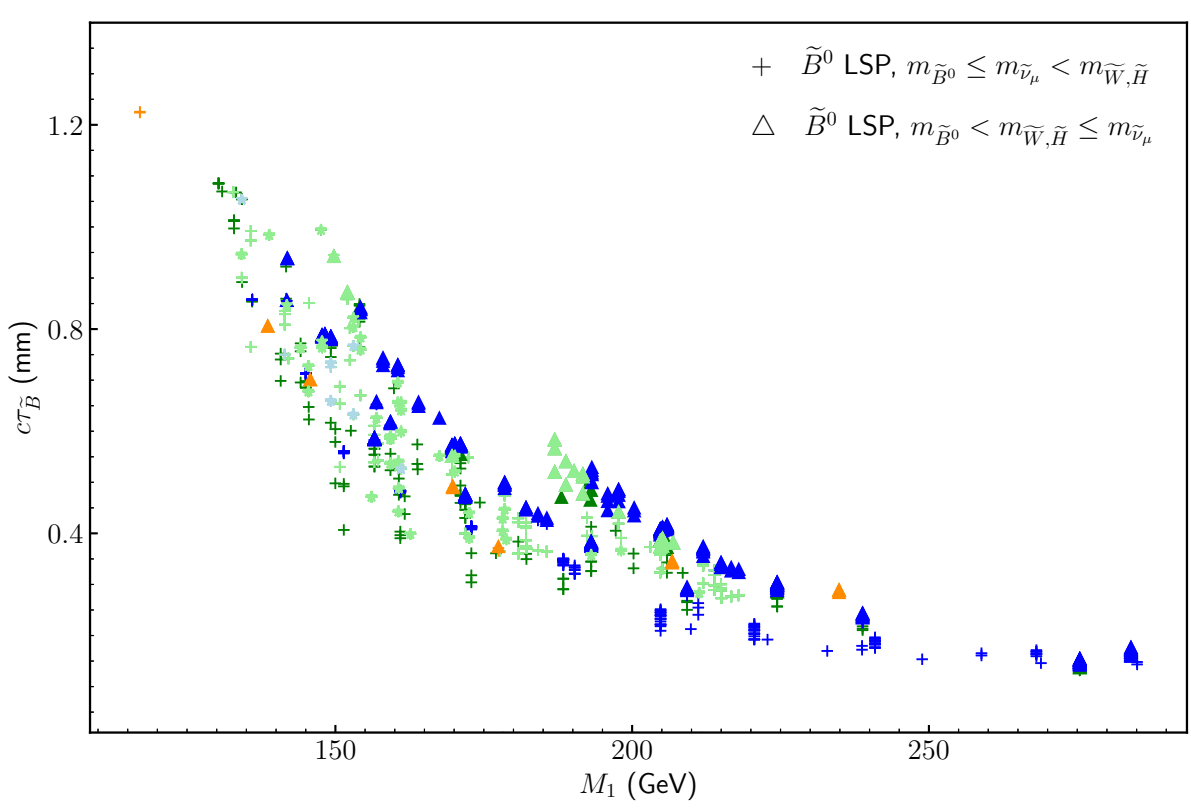

eter points corresponding to the light-green stars, on the other hand, $\tilde{\mu}_{R}$ LSP gives rise to the displaced-lepton signature. The sensitivity of the current search [102] is limited by the requirement on the transverse impact parameter, $\left|d_{0}\right|>3 \mathrm{~mm}$, which makes it insensitive to $\tilde{\mu}_{R}$ with $c \tau \lesssim 3 \mathrm{~mm}$. As we mentioned above, the decay length of $\tilde{\mu}_{R}$ is predicted to be in the range $1.5 \mathrm{~mm} \lesssim c \tau \lesssim 3 \mathrm{~mm}$, and thus lowering the condition on $\left|d_{0}\right|$ by a factor of $2-3$ may be sufficient to investigate the $\tilde{\mu}_{R}$ LSP scenario. We, therefore, strongly recommend the LHC experiments to seriously consider the optimization of the displacedvertex/displaced lepton searches for the sub-millimeter decay length.

Let us finally remark that RPV in the framework of other SUSY models has also been searched at the LHC, mainly in the MSSM. These searches typically assume either the presence of conventional trilinear lepton-number-violating couplings in the superpotential at tree level, $L L e^{c}$ and $L Q d^{c}$, or the presence of trilinear baryon-number-violating couplings, $d^{c} d^{c} u^{c}$. In all these cases we expect the results concerning $a_{\mu}$ in the (N)MSSM to be qualitatively different from those shown in Figs. 8, 9 and 10 from superpotential (6). The main reason is that in these plots the collider phenomenology has been taken into account, producing allowed/forbidden regions. However, the collider signals in the (N)MSSM with RPV will be generically different, as well as the corresponding dedicated LHC searches. For example, if one introduces the $L Q d^{c}$ or $d^{c} d^{c} u^{c}$ type operator, the decay of the LH muon-sneutrino is accompanied with jets, and thus its signature is totally different from that shown in Fig. 3. If we instead have $L L e^{c}$, the decay of the LH muon-sneutrino gives rise to multi-lepton signature without MET, and again it is completely different from Fig. 3.

\section{Conclusions}

The EW sector of the $\mu \nu$ SSM can account for a variety of experimental data, most importantly it can account for the long-standing discrepancy of $a_{\mu}$, while being in agreement with current searches at the LHC. The new result for the Run 1 data of the MUON G-2 experiment confirmed the deviation from the SM prediction found previously at BNL. Under the assumption that the previous experimental result on $a_{\mu}$ is uncorrelated with the new MUON G-2 result, we combined the data and obtained a new deviation from the SM prediction of $\Delta a_{\mu}=(25.1 \pm 5.9) \times 10^{-10}$, corresponding to a $4.2 \sigma$ discrepancy. We used this new result to set limits on the $\mu \nu \mathrm{SSM}$ parameter space.

We showed that the $\mu \nu \mathrm{SSM}$ can naturally produce light LH muon-sneutrinos and electroweak gauginos, that are consistent with Higgs and neutrino data as well as with flavor observables such as $B$ and $\mu$ decays. The presence of these light sparticles in the spectrum is known to enhance the chargino-sneutrino SUSY contribution to $a_{\mu}$, and thus it is crucial for accommodating the discrepancy between experimental and SM values. In addition, we showed that the presence of light RH smuons increasing the neutralino-smuon contribution is helpful to obtain larger values for $a_{\mu}$. We found large regions of the parameter space with these characteristics.

We applied the constraints from LHC searches on the solutions obtained. The latter have a rich collider phenomenology with the possibilities of LH muon-sneutrino, bino-like neutralino or RH smuon as LSPs. In particular, we found that multi-lepton + MET searches $[35,38,102]$ can probe regions of our scenario through the production of a chargino pair, chargino-neutralino or a neutralino pair, as well as through the production of a smuon pair, smuon-sneutrino or a sneu- 
trino pair, as shown in Figs. 3, 4, 5, 6 and 7. Overall we found significant regions of the parameter space of the $\mu \nu \mathrm{SSM}$ compatible with the world average of $a_{\mu}$ at the $2 \sigma$ level and all experimental collider data, as shown in Figs. 8 and 9. They correspond to the ranges $202 \mathrm{GeV} \lesssim M_{2} \lesssim 560 \mathrm{GeV}$, $117 \mathrm{GeV} \lesssim M_{1} \lesssim 378 \mathrm{GeV}$ and $150 \mathrm{GeV} \lesssim m_{\widetilde{e}_{2 R}} \lesssim$ $1500 \mathrm{GeV}$, where these values of the low-energy soft SUSYbreaking parameters imply that bino-like neutralino masses are in the range $114 \mathrm{GeV} \lesssim m_{\widetilde{B}^{0}} \lesssim 370 \mathrm{GeV}$, wino-like chargino/neutralino masses $200 \mathrm{GeV} \lesssim m_{\widetilde{W}} \lesssim 597 \mathrm{GeV}$, and RH smuon masses $106 \lesssim m_{\tilde{\mu}_{R}} \lesssim 1387 \mathrm{GeV}$. The corresponding LH muon-sneutrino masses are in the range $120 \lesssim m_{\widetilde{v}_{\mu}} \lesssim 540 \mathrm{GeV}$. Note that the upper bounds for $M_{1}$ and $m_{\tilde{\mu}_{R}}$ are an artifact of our scanning setup. Larger values of these parameters are possible, and they would only affect the small neutralino-smuon contribution to $a_{\mu}$. Concerning the value of $\mu$, we used $\mu \approx 380 \mathrm{GeV}, 800 \mathrm{GeV}$, corresponding to higgsino-like chargino/neutralino masses in the range $333 \mathrm{GeV} \lesssim m_{\widetilde{H}} \lesssim 878 \mathrm{GeV}$.

Finally, we discussed the implications of our results for future collider searches. As summarized in the previous paragraph, our results pin down the masses of the EW SUSY particles, and since they are predicted to be rather light, many of the allowed points in Figs. 8 and 9 can be probed in the future multi-lepton + MET searches at the (HL-)LHC. The rest of the points will be probed at a future high energy collider, such as a $100-\mathrm{TeV}$ collider, the $1-\mathrm{TeV}$ ILC, and the CLIC. Displaced-vertex searches in the future (HL-)LHC experiments offer another promising way to probe large parts of the favored parameter space and to test our scenario against the MSSM explanations, since the presence of a metastable LSP is a characteristic prediction in the $\mu \nu \mathrm{SSM}$. Many points in these figures correspond to bino LSP (crosses and triangles) or RH sneutrino LSP (stars), whose decays can be observed as a displaced decay vertex or displaced leptons, respectively. To search for these metastable particles efficiently, it is important to optimize the search strategy such that a sub-millimeter displaced vertex and $\sim 1 \mathrm{~mm}$ displaced lepton tracks can be detected. We highly encourage the ATLAS and CMS collaborations to consider this optimization seriously.

Acknowledgements The work of S.H. is supported in part by the MEINCOP Spain under contract PID2019-110058GB-C21 and in part by the AEI through the grant IFT Centro de Excelencia Severo Ochoa SEV-2016-0597. The research of E.K. and C.M. was supported by the AEI through Grants FPA2015-65929-P (MINECO/FEDER, UE), PGC2018-095161-B-I00 and IFT Centro de Excelencia Severo Ochoa SEV-2016-0597. The work of EK was funded by the IFT SEV2016-0597 and Proyecto Interno UAM-125. The work of I.L. was funded by the Norwegian Financial Mechanism 2014-2021, Grant DEC-2019/34/H/ST2/00707. The work of D.L. was supported by the Argentinian CONICET, and also acknowledges the support through PIP 11220170100154 CO. The work of N.N. was supported in part by the Grant-in-Aid for Young Scientists (No. 21K13916), Innovative Areas (No. 18H05542), and Scientific Research B (No. 20H01897). S.H.,
E.K., I.L., C.M. and D.L. also acknowledge the support of the Spanish Red Consolider MultiDark FPA2017-90566-REDC.

Data Availability Statement This manuscript has no associated data or the data will not be deposited. [Authors' comment: The datasets generated during and/or analysed during the current study are available from the corresponding author on reasonable request.]

Open Access This article is licensed under a Creative Commons Attribution 4.0 International License, which permits use, sharing, adaptation, distribution and reproduction in any medium or format, as long as you give appropriate credit to the original author(s) and the source, provide a link to the Creative Commons licence, and indicate if changes were made. The images or other third party material in this article are included in the article's Creative Commons licence, unless indicated otherwise in a credit line to the material. If material is not included in the article's Creative Commons licence and your intended use is not permitted by statutory regulation or exceeds the permitted use, you will need to obtain permission directly from the copyright holder. To view a copy of this licence, visit http://creativecomm ons.org/licenses/by/4.0/.

Funded by SCOAP ${ }^{3}$.

\section{References}

1. E. Kpatcha, I. Lara, D.E. López-Fogliani, C. Muñoz, N. Nagata, Explaining muon $g-2$ data in the $\mu \nu$ SSM. Eur. Phys. J. C 81(2), 154 (2021). arXiv: 1912.04163 [hep-ph]

2. Muon g-2 Collaboration, G. Bennett et al., Final report of the Muon e821 anomalous magnetic moment measurement at BNL. Phys. Rev. D 73, 072003 (2006). arXiv:hep-ex/0602035

3. Particle Data Group Collaboration, M. Tanabashi et al., Review of particle physics. Phys. Rev. D 98(3), 030001 (2018)

4. T. Aoyama et al., The anomalous magnetic moment of the muon in the Standard Model. Phys. Rep. 887, 1-166 (2020). arXiv:2006.04822 [hep-ph]

5. A. Keshavarzi, D. Nomura, T. Teubner, $g-2$ of charged leptons, $\alpha\left(M_{Z}^{2}\right)$, and the hyperfine splitting of muonium. Phys. Rev. D 101(1), 014029 (2020). arXiv:1911.00367 [hep-ph]

6. M. Davier, A. Hoecker, B. Malaescu, Z. Zhang, A new evaluation of the hadronic vacuum polarisation contributions to the muon anomalous magnetic moment and to $\alpha\left(\mathbf{m}_{\mathbf{Z}}^{2}\right)$. Eur. Phys. J. C 80(3), 241 (2020). arXiv:1908.00921 [hep-ph]. [Erratum: Eur. Phys. J. C 80, 410 (2020)]

7. T. Aoyama, M. Hayakawa, T. Kinoshita, M. Nio, Tenth-order QED contribution to the electron g-2 and an improved value of the fine structure constant. Phys. Rev. Lett. 109, 111807 (2012). arXiv:1205.5368 [hep-ph]

8. T. Aoyama, T. Kinoshita, M. Nio, Theory of the anomalous magnetic moment of the electron. Atoms 7(1), 28 (2019)

9. A. Czarnecki, W.J. Marciano, A. Vainshtein, Refinements in electroweak contributions to the muon anomalous magnetic moment. Phys. Rev. D 67, 073006 (2003). arXiv:hep-ph/0212229. [Erratum: Phys. Rev. D 73, 119901 (2006)]

10. C. Gnendiger, D. Stöckinger, H. Stöckinger-Kim, The electroweak contributions to $(g-2)_{\mu}$ after the Higgs boson mass measurement. Phys. Rev. D 88, 053005 (2013). arXiv: 1306.5546 [hep-ph]

11. M. Davier, A. Hoecker, B. Malaescu, Z. Zhang, Reevaluation of the hadronic vacuum polarisation contributions to the Standard Model predictions of the muon $g-2$ and $\alpha\left(m_{Z}^{2}\right)$ using newest hadronic cross-section data. Eur. Phys. J. C 77(12), 827 (2017). arXiv:1706.09436 [hep-ph] 
12. A. Keshavarzi, D. Nomura, T. Teubner, Muon $g-2$ and $\alpha\left(M_{Z}^{2}\right)$ : a new data-based analysis. Phys. Rev. D 97(11), 114025 (2018). arXiv: 1802.02995 [hep-ph]

13. G. Colangelo, M. Hoferichter, P. Stoffer, Two-pion contribution to hadronic vacuum polarization. JHEP 02, 006 (2019). arXiv:1810.00007 [hep-ph]

14. M. Hoferichter, B.-L. Hoid, B. Kubis, Three-pion contribution to hadronic vacuum polarization. JHEP 08, 137 (2019). arXiv:1907.01556 [hep-ph]

15. A. Kurz, T. Liu, P. Marquard, M. Steinhauser, Hadronic contribution to the muon anomalous magnetic moment to nextto-next-to-leading order. Phys. Lett. B 734, 144-147 (2014). arXiv: 1403.6400 [hep-ph]

16. K. Melnikov, A. Vainshtein, Hadronic light-by-light scattering contribution to the muon anomalous magnetic moment revisited. Phys. Rev. D 70, 113006 (2004). arXiv:hep-ph/0312226

17. P. Masjuan, P. Sanchez-Puertas, Pseudoscalar-pole contribution to the $\left(g_{\mu}-2\right)$ : a rational approach. Phys. Rev. D 95(5), 054026 (2017). arXiv:1701.05829 [hep-ph]

18. G. Colangelo, M. Hoferichter, M. Procura, P. Stoffer, Dispersion relation for hadronic light-by-light scattering: two-pion contributions. JHEP 04, 161 (2017). arXiv:1702.07347 [hep-ph]

19. M. Hoferichter, B.-L. Hoid, B. Kubis, S. Leupold, S.P. Schneider, Dispersion relation for hadronic light-by-light scattering: pion pole. JHEP 10, 141 (2018). arXiv:1808.04823 [hep-ph]

20. A. Gérardin, H.B. Meyer, A. Nyffeler, Lattice calculation of the pion transition form factor with $N_{f}=2+1$ Wilson quarks. Phys. Rev. D 100(3), 034520 (2019). arXiv:1903.09471 [hep-lat]

21. J. Bijnens, N. Hermansson-Truedsson, A. Rodríguez-Sánchez, Short-distance constraints for the HLbL contribution to the muon anomalous magnetic moment. Phys. Lett. B 798, 134994 (2019). arXiv:1908.03331 [hep-ph]

22. G. Colangelo, F. Hagelstein, M. Hoferichter, L. Laub, P. Stoffer, Longitudinal short-distance constraints for the hadronic light-bylight contribution to $(g-2)_{\mu}$ with large- $N_{c}$ Regge models. JHEP 03, 101 (2020). arXiv:1910.13432 [hep-ph]

23. T. Blum, N. Christ, M. Hayakawa, T. Izubuchi, L. Jin, C. Jung, C. Lehner, Hadronic light-by-light scattering contribution to the muon anomalous magnetic moment from lattice QCD. Phys. Rev. Lett. 124(13), 132002 (2020). arXiv:1911.08123 [hep-lat]

24. G. Colangelo, M. Hoferichter, A. Nyffeler, M. Passera, P. Stoffer, Remarks on higher-order hadronic corrections to the muon $\mathrm{g}-2$. Phys. Lett. B 735, 90-91 (2014). arXiv:1403.7512 [hep-ph]

25. Muon g-2 Collaboration, J. Grange et al., Muon (g-2). Technical Design Report. arXiv:1501.06858 [physics.ins-det]

26. Muon g-2 Collaboration, B. Abi et al., Measurement of the positive muon anomalous magnetic moment to $0.46 \mathrm{ppm}$. Phys. Rev. Lett. 126(14), 141801 (2021). arXiv:2104.03281 [hep-ex]

27. Muon g-2 Collaboration, T. Albahri et al., Measurement of the anomalous precession frequency of the muon in the Fermilab Muon $g-2$ Experiment. Phys. Rev. D 103(7), 072002 (2021). arXiv:2104.03247 [hep-ex]

28. S. Borsanyi et al., Leading hadronic contribution to the muon 2 magnetic moment from lattice QCD. arXiv:2002.12347 [hep-lat]

29. C. Lehner, A.S. Meyer, Consistency of hadronic vacuum polarization between lattice QCD and the R-ratio. Phys. Rev. D 101, 074515 (2020). arXiv:2003.04177 [hep-lat]

30. A. Crivellin, M. Hoferichter, C.A. Manzari, M. Montull, Hadronic vacuum polarization: $(g-2)_{\mu}$ versus global electroweak fits. Phys. Rev. Lett. 125(9), 091801 (2020). arXiv:2003.04886 [hep$\mathrm{ph}]$

31. A. Keshavarzi, W.J. Marciano, M. Passera, A. Sirlin, Muon $g-2$ and $\Delta \alpha$ connection. Phys. Rev. D 102(3), 033002 (2020). arXiv:2006.12666 [hep-ph]

32. E. de Rafael, Constraints between $\Delta \alpha_{\mathrm{had}}\left(M_{Z}^{2}\right)$ and $\left(g_{\mu}-2\right)_{\mathrm{HVP}}$. Phys. Rev. D 102(5), 056025 (2020). arXiv:2006.13880 [hep-ph]
33. D.E. López-Fogliani, C. Muñoz, Proposal for a supersymmetric standard model. Phys. Rev. Lett. 97, 041801 (2006). arXiv:hep-ph/0508297

34. D.E. Lopez-Fogliani, C. Munoz, Searching for supersymmetry: the $\mu \nu$ SSM (a short review). Eur. Phys. J. ST 229(21), 3263-3301 (2020). arXiv:2009.01380 [hep-ph]

35. ATLAS Collaboration, G. Aad et al., Search for electroweak production of charginos and sleptons decaying into final states with two leptons and missing transverse momentum in sqrts $=13$ $\mathrm{TeV} p p$ collisions using the ATLAS detector. Eur. Phys. J. C 80(2), 123 (2020). arXiv:1908.08215 [hep-ex]

36. ATLAS Collaboration, M. Aaboud et al., Search for supersymmetry in events with four or more leptons in $\sqrt{s}=13 \mathrm{TeV}$ $p p$ collisions with ATLAS. Phys. Rev. D 98(3), 032009 (2018). arXiv:1804.03602 [hep-ex]

37. ATLAS Collaboration, M. Aaboud et al., Search for electroweak production of supersymmetric particles in final states with two or three leptons at $\sqrt{s}=13 \mathrm{TeV}$ with the ATLAS detector. Eur. Phys. J. C 78(12), 995 (2018). arXiv:1803.02762 [hep-ex]

38. ATLAS Collaboration, G. Aad et al., Search for massive, longlived particles using multitrack displaced vertices or displaced lepton pairs in pp collisions at sqrts $=8 \mathrm{TeV}$ with the ATLAS detector. Phys. Rev. D 92(7), 072004 (2015). arXiv:1504.05162 [hep-ex]

39. ATLAS Collaboration, G. Aad et al., Search for displaced vertices of oppositely charged leptons from decays of long-lived particles in $p p$ collisions at $s q r t s=13 \mathrm{TeV}$ with the ATLAS detector. Phys. Lett. B 801, 135114 (2020). arXiv:1907.10037 [hep-ex]

40. CMS Collaboration, A.M. Sirunyan et al., Search for supersymmetry in final states with two oppositely charged same-flavor leptons and missing transverse momentum in proton-proton collisions at $\sqrt{s}=13 \mathrm{TeV}$. arXiv:2012.08600 [hep-ex]

41. CMS Collaboration, A.M. Sirunyan et al., Combined search for electroweak production of charginos and neutralinos in protonproton collisions at $\sqrt{s}=13 \mathrm{TeV}$. JHEP 03, 160 (2018). arXiv: 1801.03957 [hep-ex]

42. CMS Collaboration, S. Chatrchyan et al., Search in leptonic channels for heavy resonances decaying to long-lived neutral particles. JHEP 02, 085 (2013). arXiv:1211.2472 [hep-ex]

43. H.P. Nilles, Supersymmetry, supergravity and particle physics. Phys. Rep. 110, 1-162 (1984)

44. R. Barbieri, Looking beyond the standard model: the supersymmetric option. Riv. Nuovo Cim. 11N4, 1-45 (1988)

45. H.E. Haber, G.L. Kane, The search for supersymmetry: probing physics beyond the standard model. Phys. Rep. 117, 75-263 (1985)

46. J. Gunion, H.E. Haber, Higgs bosons in supersymmetric models. 1. Nucl. Phys. B 272, 1 (1986). [Erratum: Nucl. Phys. B 402, 567-569 (1993)]

47. S.P. Martin, A supersymmetry primer. arXiv:hep-ph/9709356. [Adv. Ser. Direct. High Energy Phys. 18, 1 (1998)]

48. M. Chakraborti, S. Heinemeyer, I. Saha, Improved $(g-2)_{\mu}$ measurements and supersymmetry. Eur. Phys. J. C 80(10), 984 (2020). arXiv:2006.15157 [hep-ph]

49. M. Chakraborti, S. Heinemeyer, I. Saha, Improved $(g-2)_{\mu}$ measurements and Wino/Higgsino dark matter. arXiv:2103.13403 [hep-ph]

50. M. Chakraborti, S. Heinemeyer, I. Saha, The new "MUON G-2" result and supersymmetry. arXiv:2104.03287 [hep-ph]

51. N. Escudero, D.E. López-Fogliani, C. Muñoz, R.R. de Austri, Analysis of the parameter space and spectrum of the $\mu \nu \mathrm{SSM}$. JHEP 12, 099 (2008). arXiv:0810.1507 [hep-ph]

52. P. Ghosh, I. Lara, D. E. López-Fogliani, C. Muñoz, R. Ruiz de Austri, Searching for left sneutrino LSP at the LHC. Int. J. Mod. Phys. A 33(18-19), 1850110 (2018). arXiv:1707.02471 [hep-ph] 
53. A. Brignole, L.E. Ibañez, C. Muñoz, Soft supersymmetry breaking terms from supergravity and superstring models. Adv. Ser. Direct. High Energy Phys. 18, 125-148 (1998). arXiv:hep-ph/9707209

54. M. Maniatis, The next-to-minimal supersymmetric extension of the standard model reviewed. Int. J. Mod. Phys. A 25, 3505-3602 (2010). arXiv:0906.0777 [hep-ph]

55. U. Ellwanger, C. Hugonie, A.M. Teixeira, The next-to-minimal supersymmetric standard model. Phys. Rep. 496, 1-77 (2010). arXiv:0910.1785 [hep-ph]

56. F. Capozzi, E. Di Valentino, E. Lisi, A. Marrone, A. Melchiorri, A. Palazzo, Global constraints on absolute neutrino masses and their ordering. Phys. Rev. D 95(9), 096014 (2017). arXiv:1703.04471 [hep-ph]

57. P.F. de Salas, D.V. Forero, C.A. Ternes, M. Tortola, J.W.F. Valle, Status of neutrino oscillations 2018: $3 \sigma$ hint for normal mass ordering and improved CP sensitivity. Phys. Lett. B 782, 633640 (2018). arXiv:1708.01186 [hep-ph]

58. P.F. De Salas, S. Gariazzo, O. Mena, C.A. Ternes, M. Tórtola, Neutrino mass ordering from oscillations and beyond: 2018 status and future prospects. Front. Astron. Space Sci. 5, 36 (2018). arXiv:1806.11051 [hep-ph]

59. I. Esteban, M.C. Gonzalez-Garcia, A. Hernandez-Cabezudo, M. Maltoni, T. Schwetz, Global analysis of three-flavour neutrino oscillations: synergies and tensions in the determination of $\theta_{23}, \delta_{C P}$, and the mass ordering. JHEP 01, 106 (2019). arXiv: 1811.05487 [hep-ph]

60. P. Ghosh, S. Roy, Neutrino masses and mixing, lightest neutralino decays and a solution to the $\mu$ problem in supersymmetry. JHEP 04, 069 (2009). arXiv:0812.0084 [hep-ph]

61. A. Bartl, M. Hirsch, A. Vicente, S. Liebler, W. Porod, LHC phenomenology of the $\mu \nu$ SSM. JHEP 05, 120 (2009). arXiv:0903.3596 [hep-ph]

62. J. Fidalgo, D.E. López-Fogliani, C. Muñoz, R.R. de Austri, Neutrino physics and spontaneous CP violation in the $\mu \nu$ SSM. JHEP 08, 105 (2009). arXiv:0904.3112 [hep-ph]

63. P. Ghosh, P. Dey, B. Mukhopadhyaya, S. Roy, Radiative contribution to neutrino masses and mixing in $\mu \nu$ SSM. JHEP 05, 087 (2010). arXiv:1002.2705 [hep-ph]

64. S. Liebler, W. Porod, On-shell renormalization of neutralino and chargino mass matrices in R-parity violating models-correlation between LSP decays and neutrino mixing angles revisited. Nucl. Phys. B 855, 774-800 (2012). arXiv:1106.2921 [hep-ph]

65. I. Lara, D.E. López-Fogliani, C. Muñoz, N. Nagata, H. Otono, R. Ruiz De Austri, Looking for the left sneutrino LSP with displaced-vertex searches. Phys. Rev. D 98(7), 075004 (2018). arXiv:1804.00067 [hep-ph]

66. I. Lara, D.E. López-Fogliani, C. Muñoz, Electroweak superpartners scrutinized at the LHC in events with multi-leptons. Phys. Lett. B 790, 176-183 (2019). arXiv:1810.12455 [hep-ph]

67. E. Kpatcha, D.E. López-Fogliani, C. Muñoz, N. Nagata, H. Otono, R. Ruiz De Austri, Sampling the $\mu \nu$ SSM for displaced decays of the tau left sneutrino LSP at the LHC. Eur. Phys. J. C 79(11), 934 (2019). arXiv:1907.02092 [hep-ph]

68. K.-Y. Choi, D.E. Lopez-Fogliani, C. Muñoz, R.R. de Austri, Gamma-ray detection from gravitino dark matter decay in the $\mu \nu$ SSM. JCAP 03, 028 (2010). arXiv:0906.3681 [hep-ph]

69. G.A. Gomez-Vargas, M. Fornasa, F. Zandanel, A.J. Cuesta, C. Muñoz, F. Prada, G. Yepes, CLUES on Fermi-LAT prospects for the extragalactic detection of $\mu \nu$ SSM gravitino dark matter. JCAP 02, 001 (2012). arXiv:1110.3305 [astro-ph.HE]

70. A. Albert, G. Gomez-Vargas, M. Grefe, C. Muñoz, C. Weniger et al., Search for $100 \mathrm{MeV}$ to $10 \mathrm{GeV} \gamma$-ray lines in the FermiLAT data and implications for gravitino dark matter in $\mu \nu \mathrm{SSM}$. JCAP 10, 023 (2014). arXiv:1406.3430 [astro-ph.HE]

71. G. Gomez-Vargas, D.E. Lopez-Fogliani, C. Muñoz, A.D. Perez, R.R. de Austri, Search for sharp and smooth spectral signatures of $\mu \nu$ SSM gravitino dark matter with Fermi-LAT. JCAP 1703(03), 047 (2017). arXiv: 1608.08640 [hep-ph]

72. G.A. Gómez-Vargas, D.E. López-Fogliani, C. Muñoz, A.D. Perez, $\mathrm{MeV}-\mathrm{GeV} \gamma$-ray telescopes probing gravitino LSP with coexisting axino NLSP as dark matter in the $\mu \nu$ SSM. Astropart. Phys. 125, 102506 (2021). arXiv:1911.08550 [hep-ph]

73. G.A. Gómez-Vargas, D.E. López-Fogliani, C. Muñoz, A.D. Perez, $\mathrm{MeV}-\mathrm{GeV} \gamma$-ray telescopes probing axino LSP/gravitino NLSP as dark matter in the $\mu \nu$ SSM. JCAP 2001(01), 058 (2020). arXiv:1911.03191 [hep-ph]

74. D.J.H. Chung, A.J. Long, Electroweak phase transition in the $\mu \nu$ SSM. Phys. Rev. D 81, 123531 (2010). arXiv:1004.0942 [hep$\mathrm{ph}]$

75. T. Chupp, P. Fierlinger, M. Ramsey-Musolf, J. Singh, Electric dipole moments of atoms, molecules, nuclei, and particles. Rev. Mod. Phys. 91(1), 015001 (2019). arXiv:1710.02504 [physics.atom-ph]

76. F. Gabbiani, E. Gabrielli, A. Masiero, L. Silvestrini, A complete analysis of FCNC and CP constraints in general SUSY extensions of the standard model. Nucl. Phys. B 477, 321-352 (1996). arXiv:hep-ph/9604387

77. T. Biekotter, S. Heinemeyer, C. Muñoz, Precise prediction for the Higgs-boson masses in the $\mu \nu$ SSM. Eur. Phys. J. C 78(6), 504 (2018). arXiv:1712.07475 [hep-ph]

78. T. Biekotter, S. Heinemeyer, C. Muñoz, Precise prediction for the Higgs-boson masses in the $\mu \nu \mathrm{SSM}$ with three righthanded neutrino superfields. Eur. Phys. J. C 79(8), 667 (2019). arXiv:1906.06173 [hep-ph]

79. E. Kpatcha, D.E. López-Fogliani, C. Muñoz, R. Ruiz De Austri, Impact of Higgs physics on the parameter space of the $\mu \nu \mathrm{SSM}$. Eur. Phys. J. C 80(4), 336 (2020). arXiv:1910.08062 [hep-ph]

80. T. Biekötter, munuSSM: a python package for the $\mu$-from- $\nu$ supersymmetric Standard Model. Comput. Phys. Commun. 264, 107935 (2021). arXiv:2009.12887 [hep-ph]

81. P. Ghosh, D.E. López-Fogliani, V.A. Mitsou, C. Muñoz, R. Ruiz de Austri, Probing the $\mu \nu$ SSM with light scalars, pseudoscalars and neutralinos from the decay of a SM-like Higgs boson at the LHC. JHEP 11, 102 (2014). arXiv:1410.2070 [hep-ph]

82. T. Moroi, The muon anomalous magnetic dipole moment in the minimal supersymmetric standard model. Phys. Rev. D 53, 65656575 (1996). arXiv:hep-ph/9512396. [Erratum: Phys. Rev. D 56, 4424 (1997)]

83. D.G. Cerdeno, E. Gabrielli, S. Khalil, C. Munoz, E. TorrenteLujan, Muon anomalous magnetic moment in supersymmetric scenarios with an intermediate scale and nonuniversality. Phys. Rev. D 64, 093012 (2001). arXiv:hep-ph/0104242

84. M. Endo, K. Hamaguchi, S. Iwamoto, T. Kitahara, Muon $g-2$ vs LHC Run 2 in supersymmetric models. JHEP 04, 165 (2020). arXiv:2001.11025 [hep-ph]

85. F. Feroz, M.P. Hobson, M. Bridges, MultiNest: an efficient and robust Bayesian inference tool for cosmology and particle physics. Mon. Not. R. Astron. Soc. 398, 1601-1614 (2009). arXiv:0809.3437 [astro-ph]

86. F. Staub, SARAH 4: a tool for (not only SUSY) model builders. Comput. Phys. Commun. 185, 1773 (2014). arXiv:1309.7223 [hep-ph]

87. W. Porod, SPheno, a program for calculating supersymmetric spectra, SUSY particle decays and SUSY particle production at e+ e- colliders. Comput. Phys. Commun. 153, 275 (2003). arXiv:hep-ph/0301101

88. W. Porod, F. Staub, SPheno 3.1: extensions including flavour, CPphases and models beyond the MSSM. Comput. Phys. Commun. 183, 2458-2469 (2012). arXiv:1104.1573 [hep-ph]

89. Planck Collaboration, N. Aghanim et al., Planck 2018 results. VI. Cosmological parameters. Astron. Astrophys. 641, A6 (2020). arXiv:1807.06209 [astro-ph.CO] 
90. P. Bechtle, O. Brein, S. Heinemeyer, G. Weiglein, K.E. Williams, HiggsBounds: confronting arbitrary Higgs sectors with exclusion bounds from LEP and the tevatron. Comput. Phys. Commun. 181, 138 (2010). arXiv:0811.4169 [hep-ph]

91. P. Bechtle, O. Brein, S. Heinemeyer, G. Weiglein, K.E. Williams, HiggsBounds 2.0.0: confronting neutral and charged Higgs sector predictions with exclusion bounds from LEP and the tevatron. Comput. Phys. Commun. 182, 2605 (2011). arXiv:1102.1898 [hep-ph]

92. P. Bechtle, O. Brein, S. Heinemeyer, O. Stal, T. Stefaniak, G. Weiglein, K.E. Williams, Higgs Bounds - 4: improved tests of extended Higgs sectors against exclusion bounds from LEP, the Tevatron and the LHC. Eur. Phys. J. C 74(3), 2693 (2014). arXiv: 1311.0055 [hep-ph]

93. P. Bechtle, S. Heinemeyer, O. Stal, T. Stefaniak, G. Weiglein, Applying exclusion likelihoods from LHC searches to extended Higgs sectors. Eur. Phys. J. C 75(9), 421 (2015). arXiv:1507.06706 [hep-ph]

94. P. Bechtle, D. Dercks, S. Heinemeyer, T. Klingl, T. Stefaniak, G. Weiglein, J. Wittbrodt, HiggsBounds-5: testing Higgs sectors in the LHC 13 TeV Era. Eur. Phys. J. C 80(12), 1211 (2020). arXiv:2006.06007 [hep-ph]

95. P. Bechtle, S. Heinemeyer, O. Stal, T. Stefaniak, G. Weiglein, HiggsSignals: confronting arbitrary Higgs sectors with measurements at the Tevatron and the LHC. Eur. Phys. J. C 74(2), 2711 (2014). arXiv:1305.1933 [hep-ph]

96. P. Bechtle, S. Heinemeyer, T. Klingl, T. Stefaniak, G. Weiglein, J. Wittbrodt, HiggsSignals-2: probing new physics with precision Higgs measurements in the LHC $13 \mathrm{TeV}$ era. Eur. Phys. J. C 81(2), 145 (2021). arXiv:2012.09197 [hep-ph]

97. Heavy Flavor Averaging Group Collaboration, Y. Amhis et al., Averages of B-Hadron, C-Hadron, and tau-lepton properties as of early 2012. arXiv:1207.1158 [hep-ex]

98. CMS, LHCb Collaboration, Combination of results on the rare decays $B_{(s)}^{0} \rightarrow \mu^{+} \mu^{-}$from the CMS and LHCb experiments

99. M. Santimaria, New results on theoretically clean observables in rare B-meson decays from $\mathrm{LHCb}, 2021$. Talk at LHC Seminar (2021)

100. MEG Collaboration, A.M. Baldini et al., Search for the lepton flavour violating decay $\mu^{+} \rightarrow \mathrm{e}^{+} \gamma$ with the full dataset of the MEG experiment. Eur. Phys. J. C 76(8), 434 (2016). arXiv:1605.05081 [hep-ex]

101. SINDRUM Collaboration, U. Bellgardt et al., Search for the decay $\mathrm{mu}^{+}$ightarrowe $e^{+} e^{-}$. Nucl. Phys. B 299, 1-6 (1988)

102. ATLAS Collaboration, G. Aad et al., Search for displaced leptons in $\sqrt{s}=13 \mathrm{TeV} p p$ collisions with the ATLAS detector. arXiv:2011.07812 [hep-ex]

103. ALEPH Collaboration, A. Heister et al., Search for gauge mediated SUSY breaking topologies in $e^{+} e^{-}$collisions at center-ofmass energies up to 209-GeV. Eur. Phys. J. C 25, 339-351 (2002). arXiv:hep-ex/0203024

104. OPAL Collaboration, G. Abbiendi et al., Searches for gaugemediated supersymmetry breaking topologies in e+e-collisions at LEP2. Eur. Phys. J. C 46, 307-341 (2006). arXiv:hep-ex/0507048

105. DELPHI Collaboration, J. Abdallah et al., Search for supersymmetric particles in light gravitino scenarios and sleptons NLSP. Eur. Phys. J. C 27, 153-172 (2003). arXiv:hep-ex/0303025

106. OPAL Collaboration, G. Abbiendi et al., Search for anomalous production of dilepton events with missing transverse momentum in e+ e- collisions at $\mathrm{s} * *(1 / 2)=183-\mathrm{Gev}$ to $209-\mathrm{GeV}$. Eur. Phys. J. C 32, 453-473 (2004). arXiv:hep-ex/0309014

107. DELPHI Collaboration, J. Abdallah et al., Searches for supersymmetric particles in e+ e- collisions up to $208-\mathrm{GeV}$ and interpretation of the results within the MSSM. Eur. Phys. J. C 31, 421-479 (2003). arXiv:hep-ex/0311019
108. ALEPH, DELPHI, L3, OPAL Experiments, Combined LEP GMSB Stau/Smuon/Selectron Results, 189-208 GeV. Lepsusywg/02-09.2 (2002). http://lepsusy.web.cern.ch/lepsusy/ www/gmsb_summer02/lepgmsb.html

109. ATLAS Collaboration, 'RH smuon upper limit on cross section' of 'Search for displaced leptons in $\sqrt{s}=13 \mathrm{TeV} p p$ collisions with the ATLAS detector (2020). https://doi.org/10.17182/ hepdata.98796.v1/t10

110. ATLAS Collaboration, Prospects for searches for staus, charginos and neutralinos at the high luminosity LHC with the ATLAS Detector

111. X. Cid Vidal et al., Report from Working Group 3: beyond the Standard Model physics at the HL-LHC and HE-LHC. CERN Yellow Rep. Monogr. 7, 585-865 (2019). arXiv:1812.07831 [hep$\mathrm{ph}]$

112. S. Gori, S. Jung, L.-T. Wang, J.D. Wells, Prospects for electroweakino discovery at a $100 \mathrm{TeV}$ hadron collider. JHEP 12, 108 (2014). arXiv:1410.6287 [hep-ph]

113. N. Arkani-Hamed, T. Han, M. Mangano, L.-T. Wang, Physics opportunities of a $100 \mathrm{TeV}$ proton-proton collider. Phys. Rep. 652, 1-49 (2016). arXiv:1511.06495 [hep-ph]

114. T. Golling et al., Physics at a $100 \mathrm{TeV}$ pp collider: beyond the Standard Model phenomena. arXiv:1606.00947 [hep-ph]

115. The International Linear Collider Technical Design ReportVolume 2: Physics. arXiv:1306.6352 [hep-ph]

116. A. Arbey et al., Physics at the e+ e- linear collider. Eur. Phys. J. C 75(8), 371 (2015). arXiv:1504.01726 [hep-ph]

117. K. Fujii et al., Physics case for the $250 \mathrm{GeV}$ stage of the international linear collider. arXiv:1710.07621 [hep-ex]

118. R. Franceschini et al., The CLIC potential for new physics. arXiv:1812.02093 [hep-ph]

119. M. Endo, K. Hamaguchi, S. Iwamoto, T. Kitahara, T. Moroi, Reconstructing supersymmetric contribution to muon anomalous magnetic dipole moment at ILC. Phys. Lett. B 728, 274-281 (2014). arXiv:1310.4496 [hep-ph]

120. M. Berggren, What pp SUSY limits mean for future $\mathrm{e}^{+} \mathrm{e}^{-}$colliders, in International Workshop on Future Linear Colliders (2020). arXiv:2003.12391 [hep-ph]

121. J.P. Delahaye, M. Diemoz, K. Long, B. Mansoulié, N. Pastrone, L. Rivkin, D. Schulte, A. Skrinsky, A. Wulzer, Muon colliders. arXiv:1901.06150 [physics.acc-ph]

122. K. Long, D. Lucchesi, M. Palmer, N. Pastrone, D. Schulte, V. Shiltsev, Muon colliders to expand frontiers of particle physics. Nat. Phys.17(3), 289-292 (2021). arXiv:2007.15684 [physics.acc-ph]

123. H.A. Ali et al., The Muon Smasher's Guide. arXiv:2103.14043 [hep-ph]

124. R. Capdevilla, D. Curtin, Y. Kahn, G. Krnjaic, A guaranteed discovery at future muon colliders. arXiv:2006.16277 [hep-ph]

125. D. Buttazzo, P. Paradisi, Probing the muon g-2 anomaly at a Muon collider. arXiv:2012.02769 [hep-ph]

126. W. Yin, M. Yamaguchi, Muon $g-2$ at multi-TeV muon collider. arXiv:2012.03928 [hep-ph]

127. R. Capdevilla, D. Curtin, Y. Kahn, G. Krnjaic, A no-lose theorem for discovering the new physics of $(g-2)_{\mu}$ at muon colliders. arXiv:2101.10334 [hep-ph]

128. R. Capdevilla, F. Meloni, R. Simoniello, J. Zurita, Hunting wino and higgsino dark matter at the muon collider with disappearing tracks. arXiv:2102.11292 [hep-ph]

129. T.T. Yanagida, N. Yokozaki, Muon $\mathrm{g}-2$ in MSSM gauge mediation revisited. Phys. Lett. B 772, 409-414 (2017). arXiv:1704.00711 [hep-ph]

130. M. Endo, K. Hamaguchi, S. Iwamoto, K. Yanagi, Probing minimal SUSY scenarios in the light of muon $g-2$ and dark matter. JHEP 06, 031 (2017). arXiv:1704.05287 [hep-ph]

131. K. Hagiwara, K. Ma, S. Mukhopadhyay, Closing in on the chargino contribution to the muon g-2 in the MSSM: cur- 
rent LHC constraints. Phys. Rev. D 97(5), 055035 (2018). arXiv:1706.09313 [hep-ph]

132. E. Bagnaschi et al., Likelihood analysis of the pMSSM11 in Light of LHC 13-TeV data. Eur. Phys. J. C 78(3), 256 (2018). arXiv:1710.11091 [hep-ph]

133. J.C. Costa et al., Likelihood analysis of the sub-GUT MSSM in light of LHC 13-TeV data. Eur. Phys. J. C 78(2), 158 (2018). arXiv: 1711.00458 [hep-ph]

134. G. Bhattacharyya, T.T. Yanagida, N. Yokozaki, An extended gauge mediation for muon $(g-2)$ explanation. Phys. Lett. B 784, 118-121 (2018). arXiv:1805.01607 [hep-ph]

135. M.E. Gómez, S. Lola, R. Ruiz De Austri, Q. Shafi, Dark matter, sparticle spectroscopy and muon $(g-2)$ in $S U(4)_{c} \times S U(2)_{L} \times$ $S U(2)_{R}$. JHEP 10, 062 (2018). arXiv: 1806.06220 [hep-ph]

136. B. Dutta, Y. Mimura, Electron $g-2$ with flavor violation in MSSM. Phys. Lett. B 790, 563-567 (2019). arXiv:1811.10209 [hep-ph]

137. P. Cox, C. Han, T.T. Yanagida, N. Yokozaki, Gaugino mediation scenarios for muon $g-2$ and dark matter. JHEP 08, 097 (2019). arXiv:1811.12699 [hep-ph]
138. M. Ibe, M. Suzuki, T.T. Yanagida, N. Yokozaki, Muon $g-2$ in split-family SUSY in light of LHC Run II. Eur. Phys. J. C 79(8), 688 (2019). arXiv:1903.12433 [hep-ph]

139. M. Endo, W. Yin, Explaining electron and muon $g-2$ anomaly in SUSY without lepton-flavor mixings. JHEP 08, 122 (2019). arXiv: 1906.08768 [hep-ph]

140. M. Badziak, K. Sakurai, Explanation of electron and muon $\mathrm{g}-2$ anomalies in the MSSM. JHEP 10, 024 (2019). arXiv:1908.03607 [hep-ph]

141. M. Abdughani, K.-I. Hikasa, L. Wu, J.M. Yang, J. Zhao, Testing electroweak SUSY for muon $g-2$ and dark matter at the LHC and beyond. JHEP 11, 095 (2019). arXiv:1909.07792 [hep-ph]

142. T.T. Yanagida, W. Yin, N. Yokozaki, Muon $g-2$ in Higgs-anomaly mediation. JHEP 06, 154 (2020). arXiv:2001.02672 [hep-ph]

143. ATLAS Collaboration, G. Aad et al., The ATLAS Inner Detector commissioning and calibration. Eur. Phys. J. C 70, 787-821 (2010). arXiv:1004.5293 [physics.ins-det] 